Comparative Population Studies

Selected Articles in German Vol. 41 (2016): 3-34

(Erstveröffentlichung: 22.08.2017)

\title{
Der registergestützte Zensus in Deutschland: Historische Einordnung und Bedeutung für die Bevölkerungswissenschaft
}

\author{
Rembrandt Scholz, Michaela Kreyenfeld
}

Zusammenfassung: Im Jahr 2011 führte Deutschland nach mehr als zwanzigjähriger Pause wieder einen Zensus durch. Die Vorgaben der Vereinten Nationen, nach der Länder mindestens alle zehn Jahre eine Volkszählung veranlassen sollten, waren längst überschritten und von Seiten der Bevölkerungswissenschaft wurde schon länger eine neue Zählung gefordert, um die Berechnung demografischer Indikatoren wieder auf eine solide Basis stellen zu können. Mit dem Zensus 2011 kam Deutschland der Forderung einer aktuellen Zählung nach und beschritt zugleich mit einem registergestützten Zensus einen neuen Weg. Im Gegensatz zu den skandinavischen Ländern, die auf eine lange Tradition der Registerdatenanalysen und der Verknüpfung von Registerdaten zurückblicken können, stellte in Deutschland, wo die Zusammenführung von Registern durch die gegebenen rechtlichen Rahmenbedingungen stark eingeschränkt ist, ein registergestützter Zensus ein ambitioniertes Projekt dar. Dieser Beitrag diskutiert nach einer historischen Einordnung die Bedeutung des Zensus 2011 für die Generierung zentraler demografischer Daten. Insbesondere werden die fortgeschriebenen Ergebnisse des alten Zensus 1987 mit den Ergebnissen des Zensus 2011 verglichen, um mögliche systematische Fehlerquellen zu eruieren, welche die demografischen Indikatoren und Analysen verzerren.

Schlagwörter: Demografische Kennziffern · Registerdaten · Zensus

\section{$1 \quad$ Einleitung}

Die Bedeutung des Zensus für die demografische Forschung ist unumstritten. Der Zensus liefert Schätzungen zum Bevölkerungsbestand nach Alter, Geschlecht und Region. Diese Informationen fließen in die Berechnungen von demografischen Maßzahlen, wie den Mortalitäts-, Migrations-, Nuptialitäts- und Fertilitätsziffern, ein. Zudem beeinflussen die Zensusdaten unmittelbar die Ergebnisse von amtlichen und nicht-amtlichen Befragungsdaten. Der Zensus gibt den Auswahlrahmen für die Stichprobenziehung des Mikrozensus vor, welcher wiederum den Hochrechnungs- 
rahmen für sozialwissenschaftliche Befragungsdaten darstellt. Die Bedeutung des Zensus geht jedoch weit über diese enge demografische und sozialwissenschaftliche Relevanz hinaus. Zum einen liefert der Zensus die räumlich gegliederte Einwohnerzahl, die für die Planung kommunaler Infrastruktur essenziell ist. Zum anderen werden auf Grundlage der Zensusergebnisse der Länderfinanzausgleich und der kommunale Finanzausgleich festgesetzt, nach dem die Umverteilung der öffentlichen Mittel zwischen den einzelnen Bundesländern und den Kommunen erfolgt. Zensusergebnisse sind ebenfalls für die Bestimmung der Wahlkreise für die Bundes- und Landtagswahlen, für die Sitzverteilung in Gemeinde-, Kreis- und Landräten und in gewissem Maße auch für die Sitzverteilung im Bundesrat relevant. Der deutsche Zensus ist damit, ähnlich wie die Zensen anderer Länder, weniger eine Datenerhebung für die Zwecke demografischer und sozialwissenschaftlicher Analysen, sondern vor allem ein Politikum oder wie es Prewitt (2003: 1) formuliert: „... census is a drama at the very center of our political life."

Die politische Dimension des Zensus dürfte sich in kaum einem anderen Land stärker manifestiert haben als in Deutschland. Gezeichnet von den Auseinandersetzungen um den Volkszählungsboykott 1983 und die Verschiebung der Zählung in das Jahr 1987 setzte sich Deutschland mehr als eine Dekade später dafür ein, dass die Leitlinien der Europäischen Gemeinschaft zur Durchführung eines Zensus im Jahr 2001 unverbindlich blieben (Grohmann 2011). Deutschland war das einzige EU-Land, welches diese Unverbindlichkeit nutzte und zur Jahrtausendwende keinen Zensus durchführte. Im Jahr 2008 ratifizierte die EU eine neue verbindliche Durchführungsverordnung zum Zensus 2010/11, der sich diesmal auch Deutschland anschloss. Deutschland entschied sich, im Unterschied zu den bisherigen „traditionellen“ Zensen, für einen registergestützten Zensus. Dieser Schritt war angesichts der Erfahrung mit der Volkszählung 1987 verständlich, dennoch beachtlich, da in Deutschland, anders als in den skandinavischen Ländern, wo standardmäßig Registerdaten für Zensuszwecke genutzt werden, kein zentrales Bevölkerungsregister besteht und dessen Etablierung bis heute nicht ernsthaft zur Diskussion steht. Auch eine einheitliche Identifikationsnummer für Personen wie z.B. eine Sozialversicherungsnummer, die in den skandinavischen Ländern genutzt wird, um die verschiedenen Register zu verbinden, existiert in Deutschland bis heute nicht. ${ }^{1}$ Dazu kam eine rechtliche Situation, festgelegt in $\S 21$ des Bundesstatistikgesetzes, welches strenge Auflagen bei der Verknüpfung der Register der Bundesstatistik vorsah (siehe BstatG (1987) und ZensG (2011)).

Ziel dieses Beitrags ist es, den registergestützten Zensus 2011 aus Sicht der demografischen Forschung zu diskutieren. Nach einer historischen Einordnung des Zensus 2011 (Abschnitt 1) versuchen wir in Abschnitt 2 die Genauigkeit der Populationszahlen des Zensus 2011 zu eruieren. Abschnitt 3 befasst sich mit den sys-

2007 wurde eine einheitliche Steueridentifikationsnummer eingeführt, die bislang jedoch keine Bedeutung für die systematische Zusammenführung der Registerdaten hat. Allerdings wurden im Zuge der Einführung der Steueridentifikationsnummer Korrekturen und Vervollständigungen von Adressen in den Einwohnermelderegistern durchgeführt. 
tematischen Fehlerquellen der Fortschreibung und der Bedeutung des Zensus für demografische Kennziffern. Im abschließenden Abschnitt 4 setzen wir uns kritisch mit der Frage auseinander, welchen Gewinn der Zensus 2011 für die Bevölkerungsforschung gehabt hat und welche Potenziale möglicherweise ungenutzt blieben.

\section{Volkszählung in Deutschland: Eine historische Einordnung}

\subsection{Die Entwicklung des modernen Zensus in Deutschland bis 1939}

Zensusdaten sind neben Kirchenbüchern seit jeher die zentrale Basis für demografische Analysen. Dieser Umstand kann nicht darüber hinwegtäuschen, dass nicht das genuine Interesse an demografischen oder sozialen Prozessen hinter der Entwicklung des modernen Zensus stand. Die Geschichte der statistischen Erfassung von Bevölkerung wie auch die Erhebung ausgewählter Strukturmerkmale der Bevölkerung sind unmittelbar mit der Entwicklung der öffentlichen Verwaltung verbunden und können dementsprechend kaum von den Interessen der politischen Akteure getrennt werden. Zunächst galt das Augenmerk der Erhebung der Größe der Bevölkerung, der Anzahl der Steuerzahler und der wehrfähigen Bevölkerung. Erst später wurden auch die Haushaltsstruktur sowie Merkmale der Haushaltsmitglieder wie Beruf, Bildung, Familienstand oder Staatsangehörigkeit in den Zensusdaten erfasst (Gehrmann 2009; Grohmann 2000, 2011; Rothenbacher 1997).

Volkszählungen im Gebiet des heutigen Deutschlands gibt es seit dem Mittelalter. Allerdings wurden die ersten Zählungen zumeist nur durch einzelne Städte durchgeführt. Systematische Zählungen für ganze Regionen fanden, mit unterschiedlicher Zielsetzung und Regelmäßigkeit, seit dem Beginn des 19. Jahrhunderts statt. Im Zentrum des Interesses standen die Feststellung der Bevölkerung am Wohnsitz (de jure) und später dann die tatsächlich ortsanwesende Population (de facto) zu einem Zeitpunkt (Gehrmann 2009). Demografische Ereignisse wie Geburt, Heirat und Tod wurden lange Zeit vornehmlich durch die Kirchen erfasst. Im Zuge der Reformation wurden zudem von den Kirchen so genannte protestantische "Seelenregister" oder "Martinilisten" und katholische "Status animarum" geführt, aus denen sich teilweise die Zusammensetzung der Haushalte bzw. die Familien rekonstruieren lassen. Diese Zählungen wurden von Geistlichen in vielen Regionen vorgenommen und sind, wenn auch sehr bruchstückhaft, bis heute erhalten (siehe bspw. Baten/Szołtysek 2014).

Anfänglich waren es in erster Linie Städte, die in unregelmäßigen Abständen Zählungen durchführten. Mit dem Wiener Kongress und der Gründung des Deutschen Zollvereins wurden jedoch systematische, einheitliche und regelmäßige Zählungen zur Erhebung der Bevölkerung vorgenommen (Grohmann 2000; Zahn 
1900). ${ }^{2}$ Der Hintergrund für diese Entwicklung war vor allem, dass der Beitritt der einzelnen Staaten zum deutschen Zollverein eine Kostenbeteiligung der Mitglieder an den Militärausgaben vorsah, welche entsprechend dem Bevölkerungsanteil proportional zu den jeweiligen Staaten berechnet wurde. Damit ergab sich für die Mitgliedsstaaten des Zollvereins die Notwendigkeit regelmäßig Zählungen durchzuführen, um den Bevölkerungsbestand feststellen zu können. Darüber hinaus dienten die Erhebungen dazu Steuerlisten zu erstellen, um die Kopf-, Grundbesitz-, Gewerbe-, Einkommen-, Feuerstellen-, Haushalt-, Armen-, Kriegs- und nicht zuletzt die Kirchensteuer zu erheben. Eine Beteiligung aller Einwohner an den Gemeinkosten, wie der Waisen- und Armenfürsorge, wurde über Bürgerlisten bzw. Einwohnerlisten realisiert.

Die Etablierung des Vereins für deutsche Statistik 1846 und des Statistischen Zentralbureaus in Frankfurt nach 1848 gaben wichtige weitere Impulse für die Entwicklung der unabhängigen Statistik mit einheitlichen Standards. Mit dem Zensus 1864 wurde bspw. der Haushalt als Erhebungseinheit eingeführt (Gehrmann 2009). Die letzte Volkszählung des Deutschen Zollvereins von 1867 gilt zudem als Vorbild für alle nachfolgenden Volkszählungen in Deutschland und anderen europäischen Staaten. Es war eine Volkszählung mit Haushaltslisten, die die ortsanwesende $D e$ facto-Bevölkerung und die zum Stichtag nicht anwesenden Einwohner umfasste. ${ }^{3}$

Die Trennung der Aufgaben von Kirche und Staat und die Gründung des Deutschen Kaiserreichs 1871 haben den amtlichen Volkszählungen in Deutschland einheitliche Qualitätsstandards und eine einheitliche Systematik gegeben (Miche/ 1985). Mit diesem Prozess ging auch einher, dass nicht mehr die Kirchen, sondern nunmehr auch staatliche Institutionen demografische Ereignisse wie Geburt, Tod und Eheschließung dokumentierten und diese Informationen an die statistischen Ämter weitergaben. Die amtliche Statistik wurde als unabhängiger Teil der Verwaltung entwickelt und musste für die Erfüllung vielfältiger Verwaltungsaufgaben die Datengrundlagen zur Verfügung stellen. Ab 1871 wurde in regelmäßigen Abständen von vier bzw. fünf Jahren in allen Regionen Deutschlands eine Zählung durchgeführt, deren Abstände ab 1900 auf zehn Jahre ausgeweitet werden sollten (für einen Überblick über die Zensen in Deutschland siehe Tabelle A1 im Anhang).

2 Mit dem Bundesmatrikel 1818 wurden die Impulse für eine einheitliche Bemessung der Kosten des Landsturms gesetzt. 1834 wurde von Preußen, Bayern, Hessen-Darmstadt, Hessen-Kassel und Württemberg der Deutsche Zollverein gegründet und Zählungen wurden alle drei Jahre durchgeführt (Gehrmann 2009). Sie erstreckten sich zunächst über vier Wochen, ab 1843 über drei Tage und ab 1858 auf einen Bestand am Stichtag. Es handelte sich um die Zollabrechnungsbevölkerung, welche oft mit der Erhebung der polizeilichen Melderegister oder Steuerlisten verbunden wurde. Die Zählungen wurden zudem teilweise gekoppelt mit Gebäudezählungen, Viehzählungen und der Erhebung der Personen im Wehrdienstalter.

3 Die ältesten bis in die heutige Zeit vollständig erhaltenen Volkszählungsunterlagen in Deutschland sind jene des Herzogtums Mecklenburg-Schwerin (Großherzogliches Statistisches Amt 1898; Manke 2005; Scholz 2013). Für das Herzogtum Mecklenburg-Schwerin existieren Urbelege der handschriftlich ausgefüllten Erhebungsbögen, die Haushaltslisten nach den Gemeinden mit Angaben zu Namen, Alter, Geschlecht, Familienstand, Konfession und Tätigkeit/Beruf des Haushaltsvorstandes und weiteren Merkmalen für die Zählungen der Jahre 1819, 1867, 1890 (teilweise) und 1900 enthalten. 
Durch den ersten Weltkrieg wurden die Zählungsmodi unterbrochen, und es wurden in den Kriegsjahren nur Notzählungen durchgeführt, die vor allem zur Verwaltung der Notversorgung der Bevölkerung mit Nahrungsmitteln dienten. Ein unrühmliches Kapitel nimmt in den folgenden Jahren die für 1938 geplante und 1939 durchgeführte Volkszählung ein (siehe dazu ausführlich Wietog 2001). Zwar gehen Historiker mittlerweile davon aus, dass die Volkszählung 1939 nicht für die Identifikation und Deportation von Juden verwendet wurde, was allerdings in erster Linie darauf zurückgeführt wird, dass die Daten der Zählung relativ spät aufbereitet worden waren und andere Quellen zur Identifikation von jüdischer Abstammung und Glauben zur Verfügung standen. Allerdings weist Ehmer (2013: 74) darauf hin, dass von Seiten des Statistischen Reichsamtes durchaus Publikationen existieren, in denen auf Basis der Volkszählung 1939 die Anzahl der in Deutschland verbliebenen Juden generiert und auf die "Effektivität antisemitischer Verfolgung" verwiesen wird.

\subsection{Volkszählung im getrennten Deutschland}

Nach dem zweiten Weltkrieg führten die vier Besatzungsmächte bereits im Jahr 1946 eine Zählung durch (siehe Tabelle A1 im Anhang). Bis 2011 sollte es jedoch die letzte einheitlich durchgeführte Volkszählung in Deutschland bleiben. Ab 1949 wurden in der amtlichen Statistik in den beiden Teilen Deutschlands getrennte Wege beschritten. Während in Westdeutschland ein föderales System der Verwaltung und Statistik auf Länderebene eingeführt wurde, setzte die DDR das zentrale Statistiksystem fort (Fischer 1994; Oettel 2006; Statistisches Bundesamt 1999). In der DDR erfolgte die Erhebung von Volkszählungsdaten dezentral, die Aufbereitung zentral in der Zentralverwaltung für Statistik (später "Statistisches Amt der DDR“). Die regionalen Daten der Bezirke, Kreise und Gemeinden wurden nach zentraler Zusammenführung und Auswertung der Verwaltung wieder zur Verfügung gestellt. Seit Mitte der 1970er Jahre wurde in der DDR nach dem Vorbild der skandinavischen Länder ein zentraler Einwohnerdatenspeicher etabliert. Dieses Bevölkerungsregister umfasste alle Einwohner und war mit den Bewegungsmengen Geburt, Tod und Wanderung verbunden. Die letzte traditionell durchgeführte Volkszählung der DDR des Jahres 1981 zeigte eine nur geringfügige Abweichung des fortgeschriebenen Registers von der Stichtagsbevölkerung der Volkszählung (20.000 Personen bei 16,7 Mio. Einwohnern). Die letzte Registerzählung des zentralen Einwohnerdatenspeichers wurde am 3. Oktober 1990 durchgeführt. Die Einwohnerzahlen des Registerabzugs wurden als Anfangsbestand der Bevölkerungszahlen der "Neuen Länder“ in die nun gemeinsame bundesdeutsche Bevölkerungsstatistik eingepflegt. ${ }^{4}$

Im Gegensatz zur DDR wurden (und werden) in der Bundesrepublik die Erhebungen und die Aufbereitung der amtlichen Daten dezentral auf Ebene der Län-

4 Die Daten des Einwohnerdatenspeichers (Bevölkerungsregister) der DDR und die Fortschreibungsdaten der Bundesrepublik konnten weitgehend problemlos zusammengeführt werden, da die beiden Datensätze relativ gut vergleichbar waren. Beispielsweise waren die Einschlusskriterien von ortanwesender Bevölkerung und die Einbeziehung von ausländischen Militärangehörigen in den beiden Teilen Deutschlands gleich. 
der durchgeführt. Die Zusammenfassung der Ergebnisse auf Bundesebene erfolgt durch das Statistische Bundesamt. Allerdings existiert kein zentrales Einwohnermelderegister. ${ }^{5}$ Die Populationszahlen für die Bundesrepublik werden allein auf Basis der Volkszählungen, die in der Vergangenheit als klassische Haushaltbefragungen konzipiert waren, erhoben und mit Hilfe von Bevölkerungsfortschreibungen aktualisiert. Eine Zäsur stellt zweifelsfrei die Volkszählung 1987 dar, die nicht nur auf Grund des so genannten „Volkszählungsurteils“ verfassungsrechtliche Konsequenzen hatte (Grohmann 2000). Der Volkszählungsboykott wie auch die nach 1983 folgende Klagewelle gelten als wesentliche Gründe, warum sich die Bundesrepublik Deutschland nicht an dem EU-weit geplanten Zensus 2001 beteiligte (Eppmann 2004). Gleichwohl wurde unmittelbar nach der Volkszählung 1987 damit begonnen, konkrete Möglichkeiten eines registergestützten Zensus zu erörtern (Bierau 2001; Eppmann 2004; Eppmann/Schäfer 2006).

\subsection{Zensus 2011}

Im Jahr 2011 führte Deutschland schließlich nach mehr als zwanzigjähriger Pause einen Zensus durch. Im Unterschied zu den vorangegangenen Zensen wurde dieses Mal der Weg eines registergestützten Zensus beschritten. Für die Bestimmung der Populationszahlen wurden vor allem die Daten aus den dezentral geführten kommunalen Einwohnermelderegistern verwendet. Ein Problem stellten insbesondere die Übererfassungen (Karteileichen) und Untererfassungen (Fehlbestände) in den Einwohnermelderegistern dar, wobei die Ursachen der Fehler vielfältiger Natur sind. Neben Problemen der Erfassung von Personen mit mehreren Wohnsitzen sind Fortzüge von Personen ins Ausland eine der zentralen Fehlerquellen in den Registern. Im Gegensatz zu Binnenwanderungen, bei denen die Einwohnermeldeämter seit etwa dem Jahr $2000 \mathrm{Zu}$ - und Fortzüge automatisch untereinander abgleichen und es gesetzlich vorgeschriebene Pflichten gibt, beim Umzug eine Ummeldung bei den Ämtern vorzunehmen, unterliegen Fortzüge ins Ausland keiner „amtlichen Kontrolle" (Bucher 2014: 150). ${ }^{6}$ Auch das Verhalten und die Praxis in den einzelnen Einwohnermeldeämtern bedingt die Qualität der Register. In den Einwohnermelderegistern des Bundeslandes Rheinland-Pfalz bestehen bspw. relativ geringe Unterschiede zwischen Fortschreibung und neuen Zensusdaten, da dieses Bundesland bereits in den 1970ern ein „Iandesinternes Rückmeldeverfahren bei den Binnenwanderungen" einführte (Bucher 2014: 150). Zudem führt und pflegt Rheinland-Pfalz ein Landeseinwohnerregister. ${ }^{7}$

5 Zwar wurde die Etablierung eines zentralen Einwohnermelderegisters durch Wolfgang Schäuble (CDU) im Jahr 2008 in die Diskussion gebracht (Der Spiege/ 2008), seitdem aber nicht wieder ernsthaft aufgegriffen.

Im Jahr 2000 wurde das Verfahren sukzessive umgestellt. Seit etwa 2004 wurde das Verfahren der automatischen Abgleichung von Ab- und Anmeldungen flächendeckend angewandt.

7 Weitere Länder mit Zentralen Einwohnerregistern sind die Stadtstaaten Berlin, Hamburg und Bremen wie auch das Bundesland Thüringen. 
In einem komplexen mehrstufigen Korrekturverfahren wurde für den Zensus 2011 versucht, Fehler in den Daten, die von den Meldeämtern an die Statistik übermittelt wurden, zu bereinigen (Berg 2011; Dieh/ 2012; Miche/ 2004). ${ }^{8}$ Zur Überprüfung der Qualität der Einwohnermelderegister wurde zudem eine Haushaltsstichprobe gezogen, die u.a. dazu diente, die Informationen aus den Melderegistern durch eine mündliche Befragung abzugleichen. Allerdings wurde die Haushaltsstichprobe nur für Gemeinden mit über 10.000 Einwohnern genutzt, um die Qualität der Registerdaten zu eruieren. Begründet wurde die Entscheidung damit, dass der Zensustest gezeigt hatte, dass "es in großen Gemeinden mehr Ungenauigkeiten in den Melderegistern gab als in kleinen Gemeinden" (Statistisches Bundesamt 2015: 27). In kleinen Gemeinden wurde nur bei Unstimmigkeiten zwischen den Angaben aus den Melderegistern und den Angaben aus der Gebäude- und Wohnungszählung Korrekturen vorgenommen, sodass tendenziell weniger Fehler korrigiert wurden. Zudem bedingt das Verfahren generell, dass eine Übererfassung eher aufgedeckt werden konnte als eine Untererfassung, da bei der Untererfassung Personen nicht erfasst sein können, für welche keine Angaben zum Abgleich vorliegen und die daher nicht systematisch gesucht wurden.

Auf Grund des Fortschreibungsfehlers in den Bevölkerungszahlen wurde vermutet, dass insbesondere die Mortalitätsziffern im höheren Alter, die Sterberaten der ausländischen Population und die Fertilitätsziffern der ausländischen Bevölkerung, die auf Basis der Fortschreibung generiert wurden, verzerrt waren (Kibele et al. 2008). Entsprechend gespannt wurden die neuen Populationszahlen des registergestützten Zensus 2011 erwartet. Diese Erwartung wurde auf Grund des langen Zeitraums, der zwischen Erhebung und Veröffentlichung der Daten verstrichen ist, jedoch getrübt. Der Stichtag des Zensus war der 9. Mai 2011. Die ersten zum Teil vorläufigen und hoch aggregierten Ergebnisse standen im Mai 2013 zur Verfügung. Die ersten Daten nach Alter und Geschlecht wurden jedoch erst im Januar 2014 publiziert. Im April 2015 wurden schließlich die endgültigen Ausgangsdaten der Bevölkerungsfortschreibung des Zensus 2011 veröffentlicht. Zwischen Erhebung und Publikation der endgültigen Ergebnisse lagen damit vier Jahre. Obwohl klar war, dass die verwendeten Daten eine Nettoabweichung der Bevölkerungszahlen von etwa 1,5 Millionen zum Stichtag beinhalteten, musste entsprechend noch bis zu vier Jahre nach dem Zensus mit den Daten der Fortschreibung auf Basis der Volkszählung 1987 gerechnet werden, da den neuen Daten "ein inhaltlicher oder juristischer Vorbehalt" (Bucher 2014: 149) anhing. Auch das Statistische Bundesamt musste für die 13. koordinierte Bevölkerungsvorausberechnung und für die Sterbetafeln 2010/12 auf die endgültigen Zensusdaten warten, die nach Alter, Geschlecht und Staatsbürgerschaft sehr spät zur Verfügung standen. In internationalen demografischen Datensätzen, wie der Human Fertility Database (HFD) und der Human Morta-

8 Auf Grund des so genannten „Rückspielverbots“ wurden die vom Statistischen Amt durchgeführten Korrekturen jedoch nicht an die Einwohnermelderegister zurückgegeben, sodass die Bevölkerungszahlen des Zensus von jenen in den Einwohnermelderegistern zum Zensus-Stichtag abweichen können (Kaus/Mundil-Schwarz 2015). 
lity Database (HMD), wurden ebenfalls bis vor Kurzem noch die "alten“ Fortschreibungsergebnisse verwendet. ${ }^{9}$

\section{$3 \quad$ Wie genau sind die Populationszahlen des Zensus 2011?}

Nach der aktuellen Zählung belief sich die amtlich festgestellte Bevölkerungszahl der Bundesrepublik Deutschland am Stichtag des 9. Mai 2011 auf 80.219.695 Personen (Statistisches Bundesamt 2014c). Die Genauigkeit der Angabe der Bevölkerungszahl kann jedoch kaum darüber hinwegtäuschen, dass die Bevölkerungszahl in Deutschland - ebenso wie dies für die meisten anderen Länder auch der Fall ist (Hillygus et al. 2000: 17) - das Produkt einer komplexen Schätzung darstellt. ${ }^{10}$ Während es aus Sicht der Forschung naheliegen würde Sensitivitätsanalysen durchzuführen, um die Robustheit der Schätzung zu eruieren, verlangt die politische Dimension des Zensus eine eindeutige, amtlich festgestellte und möglichst unanfechtbare Bevölkerungszahl. Trotz (oder vermutlich gerade aufgrund) des komplexen logistisch anspruchsvollen Verfahrens und der vordergründigen Genauigkeit der Angaben werden die Zensusergebnisse besonders von jenen Gemeinden in Frage gestellt, die sich durch den Zensus benachteiligt sehen. Angriffsfläche lieferte der aktuelle Zensus vor allem durch die systematisch unterschiedliche Behandlung von kleinen und großen Gemeinden (Christensen et al. 2015; Rendtel 2015; Statistisches Bundesamt 2015).

Für die demografische Forschung stellt sich die Frage, inwiefern der Zensus 2011 die Populationszahlen präzise abbildet. Obwohl die Verfahren der Datengenerierung in diversen Publikationen des Statistischen Bundesamtes beschrieben wurden (Statistische Ämter des Bundes und der Länder 2004; Statistisches Bundesamt 2015) und in verschiedenen Kommissionen, wie der Zensuskommission und ihren Unterarbeitsgruppen, mit Vertretern der Wissenschaft im Vorfeld diskutiert wurden, sind die Möglichkeiten der Wissenschaft, die Qualität der Zensusdaten zu überprüfen, äußerst begrenzt. Aufgrund der Komplexität des Verfahrens und aufgrund der Tatsache, dass die Wissenschaft letztendlich nur die endgültigen Zahlen analysieren und das Verfahren, das diese Zahlen generiert, nur über sekundäre Quellen rekonstruieren kann, kommt die Validierung des Zensus einer "Detektivarbeit" (Rendtel 2015) gleich.

9 In der HFD (http://www.humanfertility.org/) und HMD (http://www.mortality.org/) werden demografische Daten von verschiedenen Ländern der Erde vergleichbar aufbereitet und der wissenschaftlichen Gemeinschaft und der interessierten Öffentlichkeit zum „Download" zur Verfügung gestellt. Da ein hoher Anspruch an die Vergleichbarkeit der Daten nicht nur zwischen den einzelnen Ländern, sondern auch über die Zeit gelegt wird, wird angestrebt, die durch die Fortschreibungsfehler in Deutschland entstandenen Verzerrungen über eine Rückrechnung auszugleichen. Auf Grund der relativ späten Bereitstellung der neuen Zensusergebnisse konnte erst kürzlich mit der Rückrechnung begonnen werden.

10 Eine Zensuszahl ist eine Schätzung der Bevölkerung, die von der zeitlichen Erfassung der Bewegungsmengen, also der Meldung von Geburt, Zuzug, Wegzug und Tod, abhängt. Die Bewegungsmengen, welche die Bevölkerungszahl im Register zum Stichtag beeinflussen, benötigen teilweise einen Zeitraum von bis zu einem halben Jahr, bis sie vollständig gemeldet und bearbeitet sind (Hannemann/Scholz 2009). 
Im Folgenden unternehmen wir den Versuch, die Bevölkerungszahlen des Zensus $2011 \mathrm{zu}$ „eruieren“. Eine komplette Validierung der Daten ist nur bedingt möglich, da verlässliche externe Daten fehlen (siehe jedoch Abschnitt 4.2: Externe Validierung von Zensus 2011 und Fortschreibung am Beispiel der Hochaltrigen). Auch können Sensitivitätsanalysen nicht durchgeführt werden, da hierzu ein Zugang zu den Daten der Einwohnermeldeämter und der Haushaltsbefragung nötig wäre, auf deren Basis die Bevölkerungszahlen generiert wurden. Zwar hat das Statistische Bundesamt die Haushaltsbefragung mittlerweile für wissenschaftliche Analysen zugängig gemacht und plant ebenfalls die Zensusdaten als Individualdatensatz verfügbar zu machen. Da alle Hilfsmerkmale aus Datenschutzgründen zum frühestmöglichen Zeitpunkt gelöscht wurden, ist es jedoch unmöglich, selbst wenn die Individualdaten des Zensus einmal der Wissenschaft vorliegen werden, die Berechnung der Einwohnerzahlen nachzuvollziehen.

Uns liegt zur Bewertung des Zensusergebnisses 2011 lediglich das Fortschreibungsergebnis auf der Grundlage des vorangegangenen Zensus 1987 vor. Aus Mangel an besseren Alternativen schließen wir uns im Folgenden dem Vorgehen von Bucher (2014), Rendtel (2015), Christensen et al. (2015) und Kaus/Mundil-Schwarz (2015) an, die die Bevölkerungszahlen des Zensus 2011 mit denen der Fortschreibungsergebnisse des Zensus 1987 verglichen haben. Zwar haben wir kein valides externes Maß, um die Qualität des einen oder anderen Datensatzes abzuschätzen. Jedoch können wir aus der Systematik der Abweichungen gewisse Rückschlüsse darüber ziehen, welche Faktoren es sind, die die Zensusergebnisse verzerren. Als Datenbasis dienen die vom Statistischen Bundesamt veröffentlichten Zahlen des Zensus 2011 zum 9. Mai 2011 und die Daten des Fortschreibungsbestandes des Zensus 1987 zum jeweiligen Jahresende (Statistisches Bundesamt 2013b, 2014a/b).

Im Rahmen des Zensus 2011 wurde eine Befragung durchgeführt, die $10 \%$ der Haushalte in Deutschland umfasste, um zusätzliche Informationen, die in den Registern nicht verfügbar waren, zu erhalten. Zudem diente die Stichprobe dazu Fehler in den Melderegistern zu identifizieren. Bisherige Evaluationen haben im Wesentlichen gezeigt, dass für die kleineren Gemeinden, für die Korrekturen im geringeren Umfang vorgenommen wurden, das Zensusergebnis weniger von der Fortschreibung abweicht und große Gemeinden im Durchschnitt stärkere Abweichungen zu verzeichnen haben (Bucher 2014; Christensen et al. 2015). In Abbildung 1 haben wir dieses Ergebnis in Form eines Scatterplots repliziert. Jeder der in der Grafik abgebildeten Punkte entspricht einer Gemeinde. Insgesamt sind es etwa $11.000 \mathrm{Ge}$ meinden, die in der Abbildung dargestellt sind. Die Ordinate bildet die Differenz zwischen Zensus 2011 und den „alten“ Fortschreibungsergebnissen ab. Die Abszisse umfasst die Bevölkerungszahl in der jeweiligen Gemeinde. Aus der Abbildung wird ersichtlich, dass die relative Variation für kleinere Gemeinden deutlich höher ist als für größere Gemeinden. Auch wird erkennbar, dass die Variation sprunghaft mit einer Einwohnerzahl von 10.000 Personen zurückgeht.

Abbildung 2 stellt das Ausmaß der Abweichungen zwischen Zensus 2011 und Fortschreibung systematisch für kleine und große Gemeinden gegenüber. Die Abszisse gibt die relative Differenz zwischen Zensus und Fortschreibung wieder. Auf der Ordinate ist die Anzahl der Gemeinden dargestellt. Die Abbildung zeigt, dass 
Abb. 1: Relative Differenz (in Prozent) zwischen Fortschreibung 1987 und Zensus 2011 nach der Größe der Gemeinden am 09.05.2011

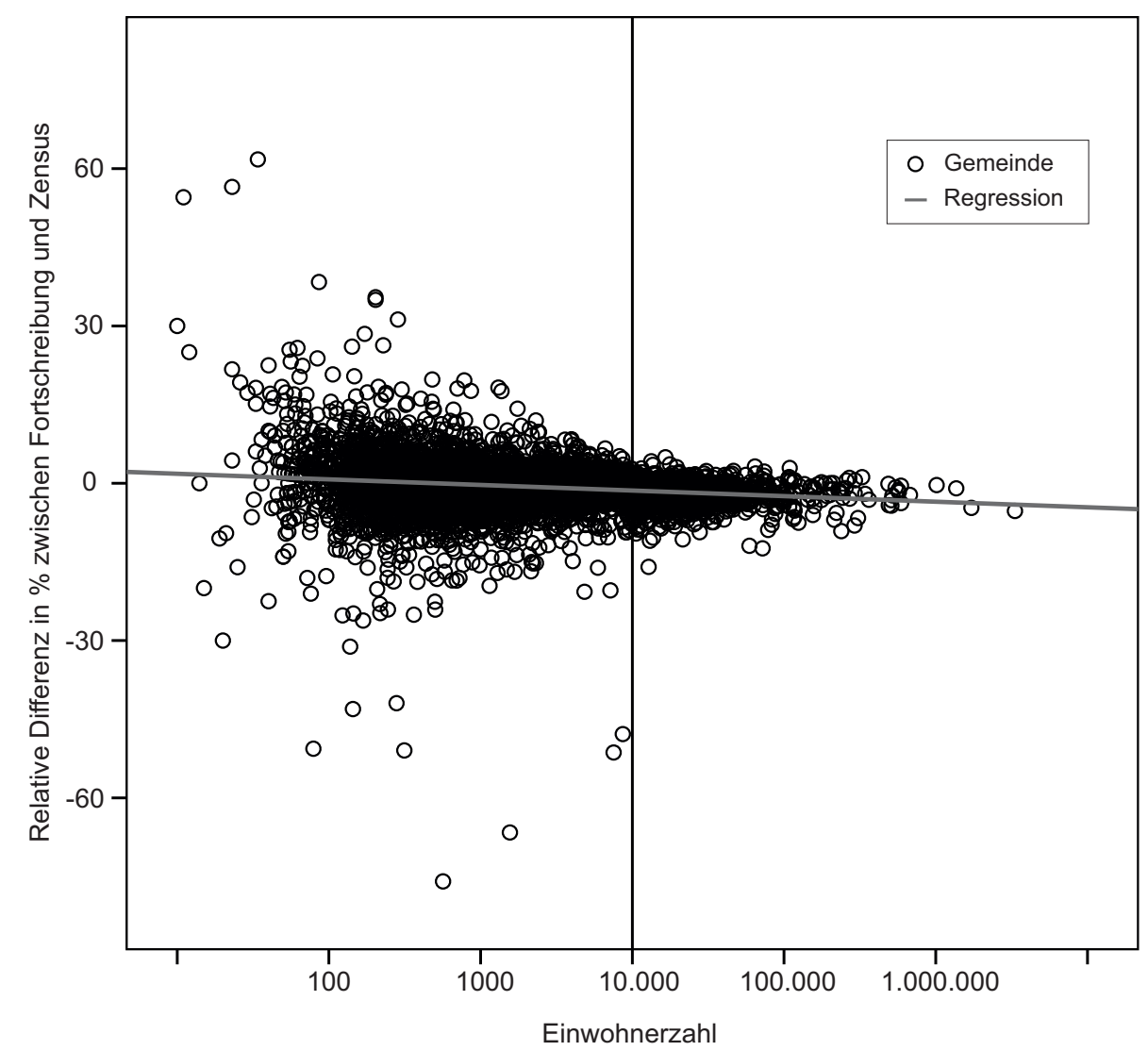

Quelle: Statistisches Bundesamt 2014a/b

große Gemeinden deutlich weniger Abweichung zu verzeichnen haben. Im Durchschnitt ergibt sich für große Gemeinden eine relative Differenz zwischen Fortschreibung und Zensus von 2,27 \%, bei den kleinen Gemeinden von 0,48 \%. ${ }^{11}$ Politisch brisant an diesem Befund ist, dass der Länderfinanzausgleich von der Bevölkerungszahl abhängig ist und kleine und größere Gemeinden unterschiedlich stark in den einzelnen Bundesländern vertreten sind. Rheinland-Pfalz hat bspw. relativ viele kleine Gemeinden; Nordrhein-Westfalen im Gegensatz dazu relativ wenige Gemeinden mit unter 10.000 Einwohnern (für eine Darstellung der Abweichungen nach Bundesländern siehe Abbildung A1 im Anhang). Dadurch, dass große und kleine Gemeinden unterschiedlich auf die Länder verteilt sind, könnte es sein, dass

11 Die Mittelwerte wurden berechnet, indem für die Populationszahlen der jeweiligen Gemeinde, laut Zensus 2011, gewichtet wurde. 
Abb. 2: Anzahl der Gemeinden (Ordinate) nach relativer Differenz zwischen Zensus 2011 und Fortschreibung 1987 (Abszisse), nach kleinen Gemeinden (unter 10.000 Einwohner) und großen Gemeinden (10.000 und mehr Einwohner), in \%

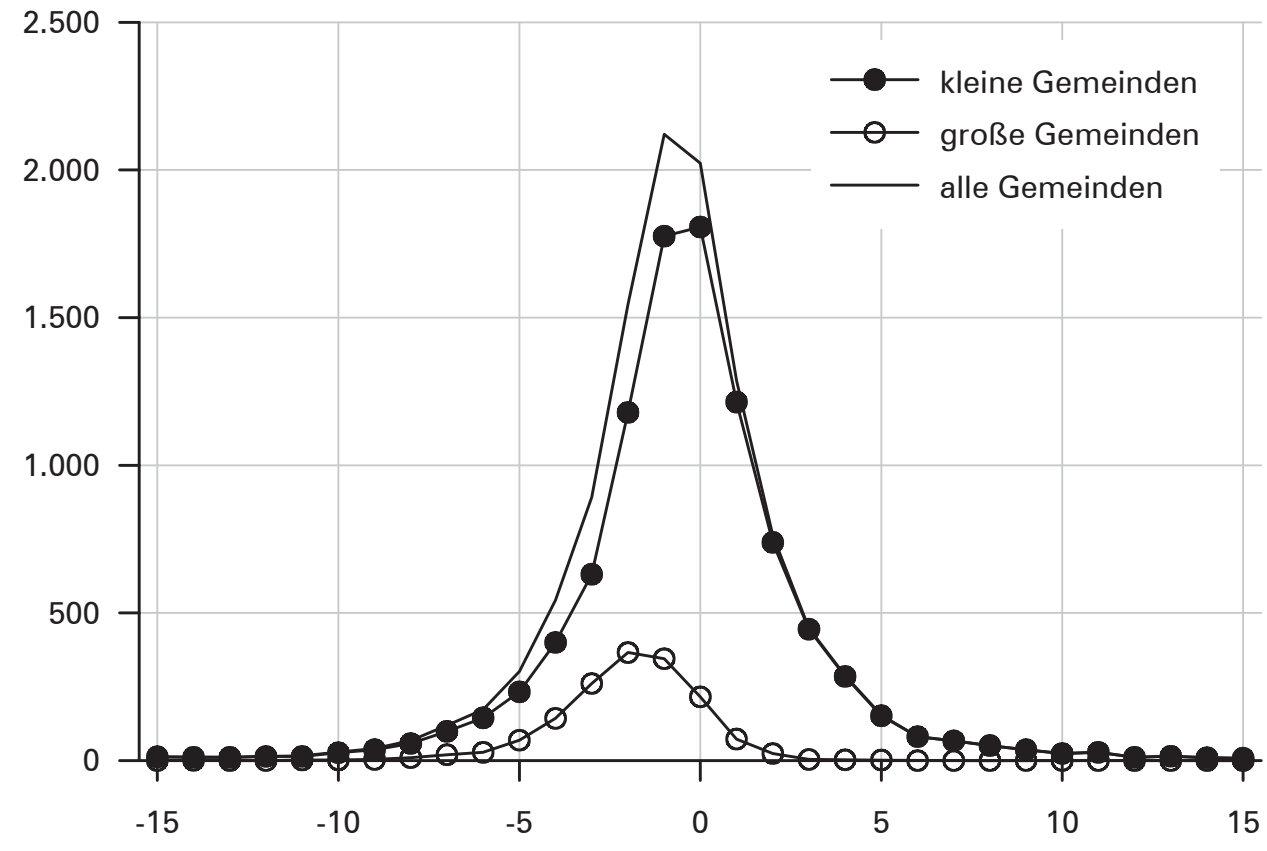

Anmerkung: Die Einteilung in große und kleine Gemeinden erfolgte nach der Einwohnerzahl der Fortschreibung.

Quelle: Statistisches Bundesamt 2013b

Bundesländer mit einem größeren Anteil von kleineren Gemeinden eine geringere Korrektur der Bevölkerungszahl durch den Zensus erfahren haben (siehe dazu ausführlich Christensen et al. 2015; Rendtel 2015). Der sprunghafte Rückgang der Variation bei 10.000 Einwohnern spricht dafür, dass die Methode einen systematischen Einfluss auf die Bevölkerungszahlen hatte. Eine Entscheidung, ob die unterschiedlichen Abweichungen zwischen Fortschreibung und Zensus nach Gemeindegröße nur an dem Verfahren liegen oder auch die Qualität der Fortschreibung eine Rolle spielt, die mitunter in kleinen Gemeinden besser sein könnte, kann an dieser Stelle nicht getroffen werden. ${ }^{12}$ Davon unabhängig ist davon auszugehen, dass durch

12 Die Altersstruktur und der Ausländeranteil sind wesentliche Aspekte, die die Qualität der Einwohnermelderegister der Gemeinden beeinflussen. Da der Ausländeranteil in den kleinen Gemeinden im Durchschnitt niedriger ist als in großen Gemeinden, sind die Register der kleinen Gemeinden weniger durch nicht dokumentierte Wegzüge von Ausländern beeinträchtigt. Zudem ist die hoch mobile Altersklasse der 20- bis 39-jährigen Personen in den kleinen Gemeinden schwächer besetzt als in großen Gemeinden (siehe Abb. A3 im Anhang). Auch dieser Aspekt dürfte die Qualität der Register der kleinen Gemeinden positiv beeinflussen. 
das angewandte Verfahren die Bevölkerung insgesamt in Deutschland etwas überschätzt wurde, da in den kleineren Gemeinden die Bevölkerungszahlen nicht nach unten korrigiert wurden. ${ }^{13}$

\section{Qualität der Bevölkerungsfortschreibung}

\subsection{Unterschiede zwischen Zensus und Fortschreibung nach Alter, Geschlecht und Staatsangehörigkeit}

Obwohl der Zensus 2011 nur ein Schätzwert der Populationszahlen für das Jahr 2011 ist, der möglicherweise die Populationszahlen der kleinen Gemeinden leicht überschätzt, ist er dennoch die einzige Messlatte, um die Qualität der Fortschreibung zu bestimmen. Mortalitätsziffern im höheren Alter konnten in der Vergangenheit nicht mehr auf Basis der Bevölkerungsfortschreibung geschätzt werden, da bekannt war, dass die Populationszahlen in der Fortschreibung im höheren Alter deutlich verzerrt waren (Kibele et al. 2008). Eine wesentliche Ursache für die Überschätzung der Populationszahlen sind Fortzüge in das Ausland, die nicht verbucht, damit in der Wanderungsstatistik nicht erfasst und in der Bevölkerungsfortschreibung somit nicht berücksichtigt wurden.

Vor diesem Hintergrund geben die Abbildungen 3a und 3b die Differenzen zwischen Zensus und Fortschreibung nach Geburtsjahr und Geschlecht für Ausländer und Deutsche wieder. Auf der Abszisse sind die Abweichungen zwischen Fortschreibung und Zensus für Männer dargestellt und auf der Ordinate für Frauen. Jeder einzelne Punkt in der Grafik gibt einen Geburtsjahrgang wieder. Je näher die Werte in der Nähe des Nullpunkts des Koordinatensystems liegen, umso besser passen Fortschreibung und Zensusergebnisse zusammen. Positive Werte bedeuten, dass der Zensus 2011 die Populationszahlen nach unten korrigiert hat. Negative Werte zeigen entsprechend eine Korrektur nach oben an. Werte auf der Diagonalen bedeuten, dass die Verzerrungen bei Frauen und Männern gleich sind (siehe auch Abbildung A2 im Anhang für eine zweidimensionale Darstellung).

Betrachtet man zunächst die Muster für Deutsche (Abb. 3a), ist bemerkenswert, dass bei den jüngeren Jahrgängen eine Korrektur nach oben vorgenommen wurde. Bspw. wurde der Jahrgang 2009 durch den neuen Zensus leicht (um 0,5\%) nach oben korrigiert. Der Jahrgang 2009 war zum Zeitpunkt des Zensus etwa zwei Jahre alt, sodass der Fortschreibungsfehler eigentlich gering sein sollte. Vermutlich erklärt sich die negative Abweichung für die jungen Geburtsjahrgänge in der nicht eindeutigen Erfassung des Merkmals Staatsangehörigkeit für Personen mit doppelter Staatsangehörigkeit. Abbildung 3a zeigt zudem eine stärker zu den Män-

13 Allerdings wurde auch nicht systematisch nach nicht registrierten Personen gesucht, was eine Unterschätzung bedingen könnte. Nach unserem Kenntnisstand gibt es keinerlei Anhaltspunkte, um das Ausmaß einer möglichen Unterschätzung durch nicht registrierte Personen einzuschätzen. Ein weiterer Grund für eine Unterschätzung der Populationszahlen im Zensus könnten noch nicht verbuchte Wanderungen und Geburten sein (siehe dazu auch Fußnote 10). 
Abb. 3a: Darstellung der relativen Abweichung des Zensus 2011 von der Fortschreibung auf Basis des Zensus 1987 bei Männern und Frauen nach Geburtsjahrgängen für Deutsche

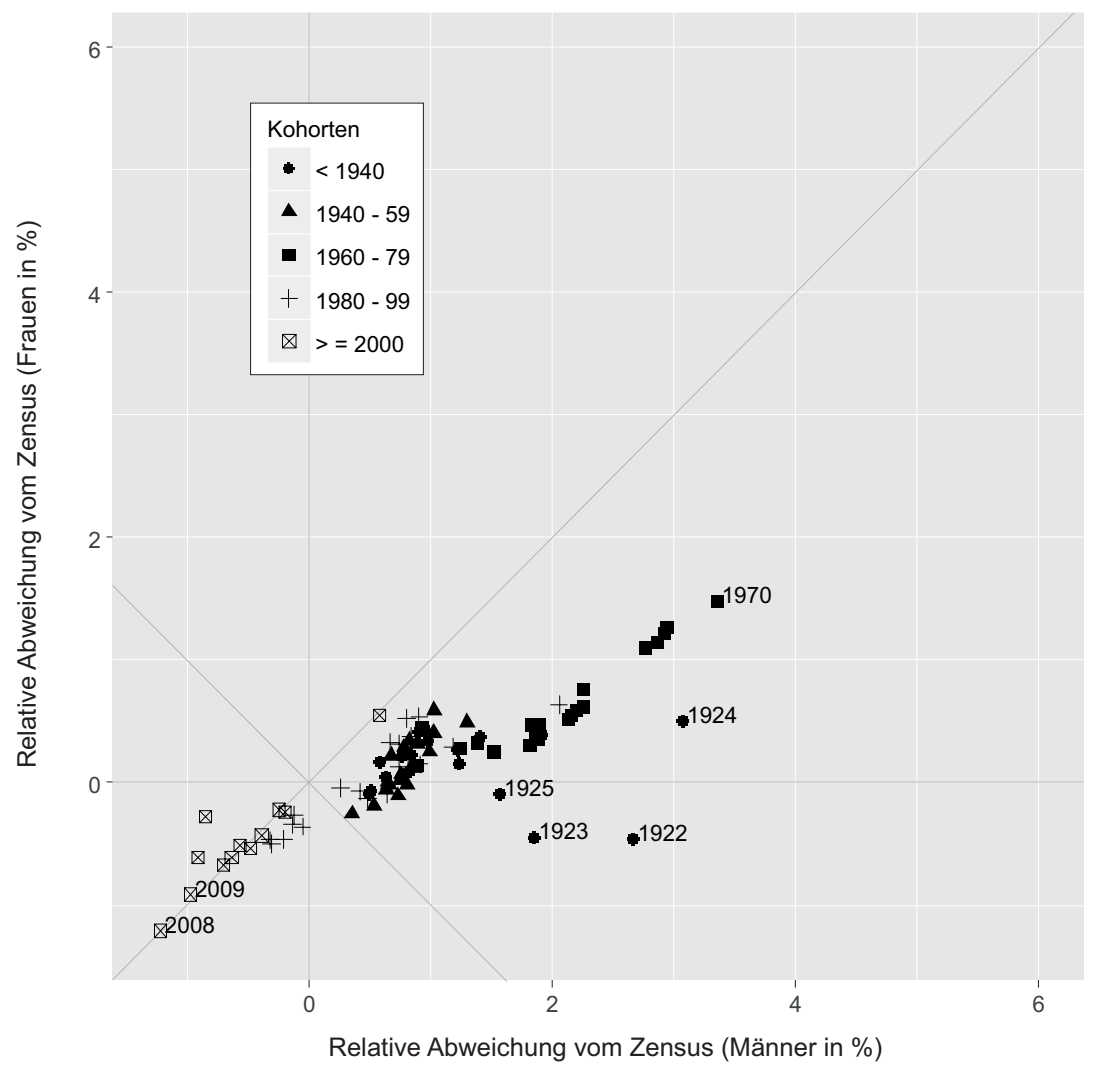

Quelle: Statistisches Bundesamt 2014a/b

nern verschobene Abweichung, welche sich nicht symmetrisch im Koordinatensystem einordnet. Für die Geschlechterunterschiede gibt es unterschiedliche Gründe. Zum einen spricht die nicht proportionale Veränderung der Abweichung der Zensusergebnisse für geschlechtsspezifische Unterschiede im Wanderungsverhalten. Zum anderen mag es sein, dass Frauen und Männer eine andere „Disziplin“ bei der behördlichen Um- und Abmeldung an den Tag legen. Des Weiteren ist auch nicht auszuschließen, dass eine unterschiedliche Korrektur nach Geschlecht bei der "Registerertüchtigung" erfolgt ist.

Abbildung $3 \mathrm{~b}$ gibt die Ergebnisse für die ausländische Population wieder. Zu beachten ist hier, dass für Ausländer eine Achse mit einem anderen Wertebereich verwendet wurde als für die deutsche Population (Abb. 3a), da die Abweichung zwischen Zensus und Fortschreibung für Ausländer deutlich größer ist als für Deutsche. Bei den Ausländern weichen die Zensus- und Fortschreibungsdaten insbesondere 
Abb. 3b: Darstellung der relativen Abweichung des Zensus von der Fortschreibung auf Basis des Zensus 1987 bei Männern und Frauen nach Geburtsjahrgängen für Ausländer

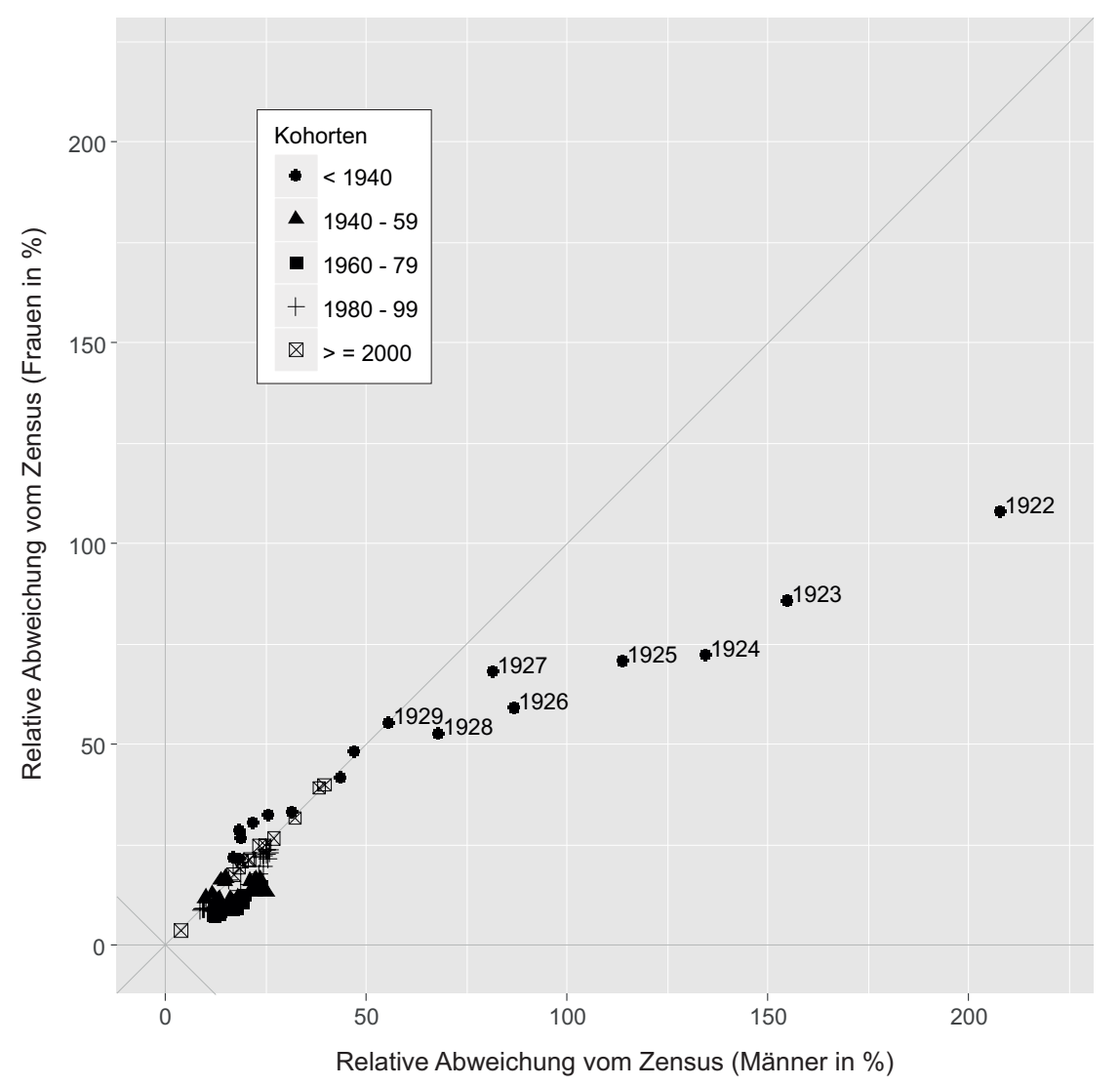

Quelle: Statistisches Bundesamt 2014a/b

im hohen Alter voneinander ab. Eine derartig auffallende Schieflage können wir bei der deutschen Population nicht feststellen. Ähnlich wie bei der deutschen Population ergeben sich auch bei der ausländischen Population bei den Männern höhere Abweichungen als bei den Frauen. Beispielsweise ergibt sich für den Geburtsjahrgang 1950 für ausländische Männer eine Abweichung zwischen Fortschreibung und Zensus 2011 von $12 \%$. Beim Geburtsjahrgang 1940 beträgt der Unterschied $17 \%$, und beim Geburtsjahrgang 1930 sind es fast 50 \%. Für die hochaltrigen Personen, die 1921 oder früher geboren worden sind, ergeben sich Abweichungen von über $600 \%$, die in der Abbildung nicht mehr dargestellt sind.

In der Zusammenschau kann man feststellen, dass die neuen Zensusergebnisse vor allem die Populationszahlen der ausländischen Bevölkerung im hohen Alter korrigiert haben. Zudem sind die Werte für Männer stärker verändert worden als jene für Frauen. Für die Fertilitäts- und Mortalitätsforschung hat der neue Zensus damit 
eine sehr unterschiedliche Bedeutung. Für die Berechnung von Fertilitätsindikatoren werden in der Regel nur die Bevölkerungszahlen von Frauen im Alter von 15 bis 49 Jahren verwendet. Für diese Gruppe ergeben sich die geringsten Abweichungen zwischen Zensus und Fortschreibung, sodass der Einfluss des neuen Zensus auf die Maße der Fertilität gering sein sollte. Verzerrt waren in erster Linie die Fertilitätsziffern nach Staatsangehörigkeit, die auf Basis der Fortschreibung generiert wurden (siehe dazu Pötzsch 2016; zur Nieden/Sommer 2016). In der Mortalitätsforschung führen die neuen Zensusergebnisse zu einer erheblichen Korrektur der Sterbeziffern im höheren Alter. Zudem dürften sich die Pflegeraten deutlich verändern (siehe dazu Tabelle A2 im Anhang). Da das Statistische Bundesamt keine Rückrechnungen zur Korrektur der Populationszahlen nach Alter anvisiert, müssen Sprünge in den Zeitreihen der demografischen Indikatoren hingenommen werden, die nach Geschlecht und vor allem nach Staatsangehörigkeit unterschiedlich ausfallen.

\subsection{Externe Validierung von Zensus 2011 und Fortschreibung am Beispiel der Hochaltrigen}

Prinzipiell liefern nur Zensus und Fortschreibung Informationen über die Bevölkerungszahlen in Deutschland. Allerdings existieren indirekte Verfahren, auf Basis derer man aus alternativen Datenquellen Populationszahlen generieren und somit die Qualität von Zensus und Fortschreibung plausibilisieren kann. Eine derartige Quelle sind die Sterbefälle im höheren Alter. Da die Sterbefälle nach Alter sich relativ sicher aus der Sterbefallstatistik herleiten lassen, kann indirekt aus dem Sterbealter auf die Anzahl der Personen nach Geburtsjahrgängen und Geschlecht in den Vorjahren geschlossen werden. Dies ist ein indirektes Verfahren, welches auf der Annahme beruht, dass in sehr hohem Alter die internationale Wanderungsaktivität gering ist. Allerdings kann diese Methode nur für jene Jahrgänge angewandt werden, die bereits verstorben sind, und muss damit auf das sehr hohe Lebensalter begrenzt bleiben. Trotz dieser Einschränkungen haben Sensitivitätsanalysen für verschiedene Länder gezeigt, dass diese Methode qualitativ bessere Bevölkerungszahlen liefert als die Daten von Fortschreibungsergebnissen (Jdanov et al. 2005).

In Abbildung 4 haben wir die Differenz der Fortschreibung von der auf Basis der Sterbefälle berechneten Anzahl der Personen im Alter 90 und älter dargestellt. Die Werte des Zensus sind mit einem Kreuz eingezeichnet. Wie aus der Abbildung ersichtlich, sind die Werte des Zensus und der auf Basis der Sterbefälle berechneten Population fast deckungsgleich, was prinzipiell für die Qualität der Zensusergebnisse im höheren Alter spricht. Im Umkehrschluss bedeutet dies, dass sich hier erneut die Schieflage der "alten“ Fortschreibung bestätigt. Im Alter 90 und älter ergeben sich im Jahr 2008 Abweichungen zwischen Fortschreibung und den „tatsächlichen“ Werten von über $30 \%$.

Abbildung 4 gibt auch die Differenz zwischen Fortschreibung und der auf Basis der Sterbefälle berechneten Population für die zurückliegenden Jahre wieder. Zu den Zeitpunkten der Volkszählungen sind die Abweichungen sehr gering, um dann mit zunehmendem Abstand zum vorangegangenen Zensus steil anzusteigen. Bei den Männern steigen die Differenzen bspw. von 0,6 \% im Zensusjahr 1987 auf etwa 
Abb. 4: $\quad$ Relative Differenz im Alter 90 Jahre und älter (in \%) zwischen der fortgeschriebenen Bevölkerung und der berechneten Bevölkerung aus den Sterbefällen bei Bezug auf die Fortschreibungsbevölkerung

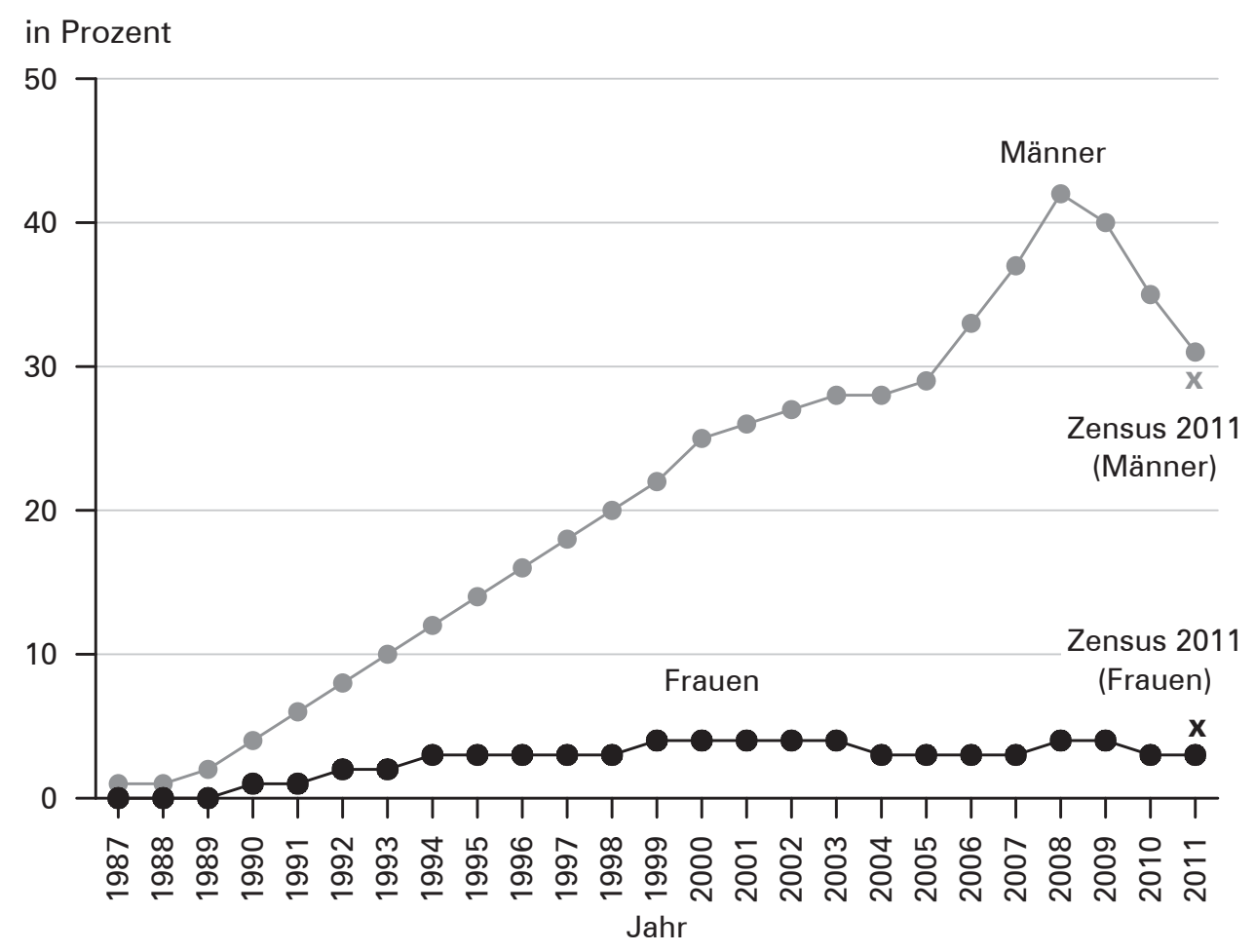

Quelle: Eigene Berechnung nach Human Mortality Database (http://www.mortality.org/)

$25 \%$ im Jahr 2011 an. Allerdings zeigt sich auch eine sehr uneinheitliche Entwicklung über die Zeit. Vor allem reduzieren sich im Jahr 2008 schlagartig die Unterschiede. Diese erratische Entwicklung erklärt sich sehr wahrscheinlich durch die Bereinigungen der Register. Zum einen wurden in den Jahren 2007 bis $2010 \mathrm{im} \mathrm{Zu-}$ sammenhang mit der Einführung der Steueridentifikationsnummer die Einwohnermelderegister bereinigt (Kaus/Mundil-Schwarz 2015). Zudem wurden im Rahmen der Zensus-Tests in den Jahren 2001 bis 2004 so genannte „Registerertüchtigungen" durchgeführt, die wahrscheinlich mit der Bereinigung des Ausländerzentralregisters in den Jahren 2001 bis 2003 in Zusammenhang stehen. Die Registerertüchtigungen dürften vor allem Registerüberhänge von Personen, die nicht mehr in Deutschland leben, aufgedeckt haben. Diese Personen wurden im Zeitraum 20012004 und 2007-2010 aus den Registern entfernt, indem sie als "Verzug unbekannt" (für Deutsche) und "Verzug in das Ausland" (für Ausländer) gekennzeichnet wurden.

Um zu verstehen, warum die Bereinigungen die Fortschreibungsergebnisse beeinflusst haben, muss man berücksichtigen, dass die Wanderungsstatistik, die für die Fortschreibung verwendet wird, auf den Einwohnermelderegistern beruht. Die 
Korrektur hat daher die Wanderungsstatistik beeinflusst. ${ }^{14}$ Problematisch ist zum einen, dass die Korrektur der Register zu einem bestimmten Zeitpunkt nach 2001 durchgeführt wurde, obwohl der Registerabgang durch nicht verbuchte Wanderungen, die wahrscheinlich in den 1990er Jahren erfolgt waren, entstanden war. Darüber hinaus ist besonders unglücklich, dass die Korrekturen nicht als solche ausgewiesen wurden, sodass für die Jahre 2008/2009 nicht mehr rekonstruiert werden kann, ob in Deutschland tatsächlich die Nettozuwanderung negativ gewesen ist oder diese allein durch die Korrektur "produziert" wurde. Da die Wanderungsstatistik auch in die Fortschreibung einfließt, wurden durch die Korrektur zudem indirekt die Bevölkerungszahlen der Fortschreibung verändert und damit die Basis zur Berechnung von demografischen Kennziffern.

\section{$5 \quad$ Fazit}

Ähnlich wie in anderen Ländern auch erfüllt der Zensus in Deutschland multiple Funktionen. Für die öffentliche Verwaltung sind der Zensus und die Fortschreibungsergebnisse Grundlage für die Planung der lokalen Infrastruktur, die Wohnstandort- und Verkehrsinfrastrukturentwicklung, Verkehrsplanung, Versorgung mit medizinischer und Pflegeinfrastruktur, Schulplanung, Bildungs- und Kulturförderung, Kostenplanung von Sozialleistungen, Energieversorgung etc. (siehe ZensG 2011 § 1). ${ }^{15}$ Für die demografische Forschung sind die Populationszahlen des Zensus zentral, da mit diesen Mortalitäts- und Fertilitätsziffern generiert werden können. Darüber hinaus kommt dem Zensus für die sozialwissenschaftliche Survey-Forschung eine wichtige Bedeutung zu. Zum einen haben die Einwohnerzahlen nach Gemeinde, Geburtsjahr und Geschlecht eine unmittelbare Relevanz bei der Ziehung von Einwohnermeldestichproben. Zum anderen liefert der Zensus den Auswahlplan, auf Basis dessen die Zählbezirke für den Mikrozensus eingeteilt werden. Dieser wiederum liefert den Rahmen für die Generierung von so genannten „post-stratification weights“, die standardmäßig in sozialwissenschaftlichen Surveys zur Verfügung gestellt werden, um die Befragungsdaten an die im Mikrozensus vorgegebene Bevölkerungsstruktur anzupassen.

Ist der Zensus über die oben genannten Aspekte hinaus von Bedeutung für die demografische und sozialwissenschaftliche Forschung? Das Potenzial des Zensus für weitergehende wissenschaftliche Forschung ergibt sich vor allem durch die gro-

$\overline{14}$ In der Beschreibung der Migrationsstatistiken heißt es dazu: „Für die Jahre 2008 und 2009 ist zu berücksichtigen, dass aufgrund der bundesweiten Einführung der persönlichen Steuer-Identifikationsnummer im Jahr 2008 umfangreiche Bereinigungen der Melderegister in diesen beiden Jahren vorgenommen wurden, die zu zahlreichen Abmeldungen von Amts wegen geführt haben. Da der Umfang dieser Bereinigungen aus den Meldungen der Meldebehörden statistisch nicht ermittelt werden kann, bleiben der tatsächliche Umfang der Fortzüge in den Jahren 2008 und 2009 sowie die Entwicklung gegenüber den Vorjahren unklar" (Bundesamt für Migration und Flüchtlinge 2015: 13).

15 Gesetz über den registergestützten Zensus im Jahre 2011 (Zensusgesetz 2011, ZensG 2011) in der Fassung vom 8. Juli 2009. 
Ben Fallzahlen, die es erlauben würden, regionalisierte Analysen durchzuführen. Da bis dato (und damit fünf Jahre nach Zensus) jedoch noch nicht geklärt ist, wie und ob regionalisierte Individualdaten des Zensus zur Verfügung gestellt werden, dürften mit zunehmender Dauer die Zensusdaten für die wissenschaftliche Forschung unattraktiver werden. Gleiches gilt für die zusätzliche Haushaltsbefragung, die ebenfalls für wissenschaftliche Mikroanalysen erst kürzlich zugängig gemacht wurde. Mittlerweile liegen aktuellere Mikrozensus-Ergebnisse vor, die ein ähnliches bzw. zum Teil größeres Potenzial für familiendemografische Fragestellungen liefern als die Haushaltsbefragung des Zensus. Die (finalen) Einwohnerzahlen nach Alter und Geschlecht, die insbesondere für die Generierung von demografischen Kennziffern relevant sind, sind mit einer Verzögerung von vier Jahren veröffentlicht worden. Zum Vergleich: In Schweden, wo systematisch Bevölkerungsregister aufgebaut wurden, werden die finalen Bevölkerungsdaten nach Alter und Geschlecht innerhalb eines Jahres publiziert.

Ein Argument für einen registergestützten Zensus war es, dass dieser kostengünstiger und effektiver durchgeführt werden kann, da die notwendigen Daten bereits in den Registern vorlägen (Bierau 2001: 335; Eppmann 2004). Die komplexen Verfahren, die notwendig waren, um die verschiedenen Register zusammenzubringen, stellt diese Vorstellung in Frage. Sicherlich ist die fehlende Transparenz und die mangelnde Nachprüfbarkeit der Zensusergebnisse teilweise den spezifischen rechtlichen Rahmenbedingungen in Deutschland geschuldet. Allerdings stellt sich durchaus die Frage, ob nicht die Möglichkeiten der Registerverknüpfung überschätzt worden sind. Selbst in Norwegen, wo über einen langen Zeitraum hinweg systematisch ein Haushaltsregister aufgebaut wurde, sind erst mit dem Zensus 2011 die Daten der Haushaltsregister für den Zensus verwendet worden (Zhang/Hendriks 2012). Es überrascht schon, dass Deutschland, das nicht auf Erfahrungen mit Haushaltsregistern zurückgreifen kann, gleich in der ersten Runde die Haushaltsinformationen über die Registerdaten in einem hoch komplexen Verfahren generiert hat (Vorndran 2004). Letztendlich sind die Möglichkeiten des registergestützten Zensus 2011 nicht genutzt worden, um ein Haushalts-, ein Bevölkerungs- (wie dies beispielsweise in der Schweiz der Fall war) oder ein Verwaltungsregister langfristig aufzubauen und zu pflegen.

Neben der gesellschaftspolitischen, demografischen und sozialwissenschaftlichen Relevanz kommt dem Zensus auch eine zentrale politische Bedeutung zu, da auf Basis der durch Zensus und Fortschreibung festgesetzten Einwohnerzahlen der Finanzausgleich zwischen Gemeinden und Ländern berechnet werden und darüber hinaus der Zuschnitt der Wahlkreise für die Bundestags- und Landtagswahlen bestimmt wird. Diese "Doppelnatur" des Zensus hat vor allem die unerwünschte $\mathrm{Ne}$ benwirkung, dass eine Kritik am Zensus, die allein wissenschaftlich begründet sein mag, sich zwangsweise immer auch der Gefahr aussetzt, für politische Zwecke von jenen instrumentalisiert zu werden, die Nachteile aus den Zensusergebnissen hinnehmen mussten. Einwohnerzahlen sind ein Politikum und eine sachliche und wissenschaftliche Auseinandersetzung mit der Generierung der Populationszahlen gestaltet sich entsprechend schwierig. Erstaunlich ist dennoch die große Vehemenz, mit der sich Politiker über die Zensuskorrekturen der Einwohnerzahlen beschweren 
(siehe für Berlin bspw. Rennefanz/Zylka 2013), während die gravierenden Mängel in den Fortschreibungsdaten in der Vergangenheit stillschweigend hingenommen wurden. Auch mit dem Zensus 2011 wurde erhebliche Energie auf die Anfechtung der Einwohnerzahlen verwendet (Statistisches Bundesamt 2016), während eine politische Auseinandersetzung über die Verbesserung der Melderegister und der Fortschreibung weitgehend ausgeblieben ist. Fakt ist, dass die Probleme, die die Populationszahlen in der Fortschreibung verzerrt haben, bis heute nicht behoben wurden. Damit ist auch für die Zukunft zu erwarten, dass die systematischen Fehler wieder im Abstand zum Zensus ansteigen werden.

\section{Danksagung}

Wir danken Harald Utne (Senior Adviser Population Statistics, Statistics Norway) für wertvolle Hinweise zur Haushaltsgenerierung im norwegischen Zensus. Gunnar Andersson (Stockholm University, Demography Unit) danken wir für Informationen zu den schwedischen Registern. Für die Unterstützung bei der Erstellung der Abbildungen 3a und 3b möchte wir uns sehr herzlich bei Rainer Walke (Max-Planck-Institut für demografische Forschung) bedanken. Für die sprachliche Durchsicht danken wir Lena Klein (Hertie School of Governance, Berlin). Für wertvolle inhaltliche Kommentare danken wir den Gutachtern und der CPOS-Redaktion.

\section{Literatur}

Baten, Jörg; Szołtysek, Mikolaj 2014: A golden age before serfdom? The human capital of Central-Eastern and Eastern Europe in the 17th-19th centuries. MPIDR Working Paper 2014-18 [http://www.demogr.mpg.de/papers/working/wp-2014-008.pdf, 25.07.2016].

BStatG 1987 (Bundesstatistikgesetz BStatG vom 22. Januar 1987): [https://www.destatis.de/DE/Methoden/Rechtsgrundlagen/Statistikbereiche/Inhalte/010_BStatG.pdf? blob=publicationFile, 25.07.2016].

Berg, Andreas; Bihler, Wolf 2011: Das Hochrechnungsverfahren zur Ermittlung der Einwohnerzahl im Zensus 2011. In: Wirtschaft und Statistik 2011/3: 230-235 [https://www. destatis.de/DE/Publikationen/WirtschaftStatistik/Zensus/HochrechnungsverfahrenEinwohnerzahl_42014.pdf?_blob=publicationFile, 25.07.2016].

Bierau, Dieter 2001: Neue Methode der Volkszählung. Der Test eines registergestützten Zensus. In: Wirtschaft und Statistik 2001/5: 333-341 [https://www.destatis.de/DE/ Publikationen/WirtschaftStatistik/Zensus/NeueMethodeVolkszaehlung_52001.pdf? blob= publicationFile, 25.07.2016].

Bucher, Hansjörg 2014: Der Zensus 2011 und seine Auswirkungen auf demographische Eckwerte. In: Europa Regional 20: 147-159 [http://nbn-resolving.de/urn:nbn:de:0168ssoar-424095, 25.07.2016].

Bundesamt für Migration und Flüchtlinge 2015: Migrationsbericht 2013. Nürnberg: BMF [https://www.bamf.de/SharedDocs/Anlagen/DE/Publikationen/Migrationsberichte/ migrationsbericht-2013. pdf; jsessionid=652E955CA8155AC5E64D85329E541331.1 cid359?__blob=publicationFile, 25.97.2016]. 
Christensen, Björn et al. 2015: Everything counts! Warum die kleinen Gemeinden die Gewinner der Zensuserhebung 2011 sind. In: Wirtschafts- und Sozialstatistisches Archiv 9,3: 215-232 [doi: 10.1007/s11943-015-0173-x].

Der Spiege/ 2008: Einwohnerdaten: Schäuble plant zentrales Bundesmelderegister. (Der Spiegel vom 27.06.2008).

Diehl, Eva-Maria 2012: Methoden der Mehrfachfallprüfung im Zensus 2011. In: Wirtschaft und Statistik 2012/4: 473-484 [https://www.zensus2011.de/SharedDocs/Downloads/DE/Publikationen/Aufsaetze Archiv/2012 06 Destatis Methoden der Mehrfachfallpruefung_im_Zensus_2011.p̄df?_blob=publicationFilę $v=14,25 . \overline{07} .2016]$.

Ehmer, Josef 2013: Bevölkerungsgeschichte und Historische Demographie 1800-2010. 2. um einen Nachtrag erweitere Auflage. München: Oldenbourg Wissenschaftsverlag.

Eppmann, Helmut 2004: Von der Volkszählung 1987 zum registergestützten Zensus 2010? In: Statistische Analysen und Studien NRW 17: 3-9 [https://www.zensus2011.de/SharedDocs/Downloads/DE/Publikationen/Aufsaetze_Archiv/2004_NRW_Von_der_Volkszaehlung zum registergestuetzten_Zensus2010.pdf? blob $=$ publication $\overline{\text { File }} \bar{\varepsilon} \mathrm{v}=11$, 25.07.2016].

Eppmann, Helmut; Schäfer, Josef 2006: Der Weg zu einem ersten registergestützten Zensus in Deutschland im Jahre 2011. In: Statistische Analysen und Studien NRW 27: 3-15 [https://www.zensus2011.de/SharedDocs/Downloads/DE/Publikationen/Aufsaetze_Archiv/2006_NRW_Der_Weg_zum_ersten_registergestuetzten_Zensus_in_

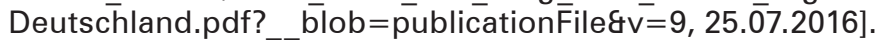

Fischer, Peter 1994: Entwicklung der amtlichen Statistik in der sowjetischen Besatzungszone Deutschlands und der DDR - 1945 bis 1990. In: Jahrbücher für Nationalökonomie und Statistik 213: 597-617.

Gehrmann, Rolf 2009: German Census-Taking Before 1871. MPIDR Working Paper 2009 023 [http://www.demogr.mpg.de/papers/working/wp-2009-023.pdf, 25.07.2016].

Grohmann, Heinz 2000: Geschichte und Zukunft der Volkszählung in Deutschland. In: Berliner Statistik (Statistische Monatsschrift) 12: 216-223.

Grohmann, Heinz 2011: Volkszählung und Mikrozensus. In: Grohmann, Heinz; Krämer, Walter; Steger, Almut (Hrsg.): Statistik in Deutschland. Berlin/Heidelberg: Springer: 207-221 [doi: 10.1007/978-3-642-15635-9_17].

Großherzogliches Statistisches Amt 1898 (Hrsg.): Statistisches Handbuch für das Großherzogthum Mecklenburg-Schwerin. Schwerin.

Hannemann, Anke; Scholz, Rembrandt D. 2009: The mortality of non-Germans in Germany: A comparison of results estimated from the Official Statistics and the Central Register of Foreigners. Rostock Center Discussion Paper 26, Rostock Center for the Study of Demographic Change [http://www.rostockerzentrum.de/content/publikationen/rz_diskussionpapier_26.pdf, 25.07.2016].

Hillygus, D. Sunshine et al. 2000: The Hard Count. The Political and Social Challenges of Census Mobilization. New York: Sage.

Jdanov, Dmitri A.; Scholz, Rembrandt D.; Shkolnikov, Vladimir M. 2005: Official population statistics and the Human Mortality Database estimates of populations aged $80+$ in Germany and nine other European countries. In: Demographic Research 13: $335-$ 362 [doi: 10.4054/DemRes.2005.13.14].

Kaus, Wolfhard; Mundil-Schwarz, Rabea 2015: Die Ermittlung der Einwohnerzahlen und der demografischen Strukturen nach dem Zensus 2011. In: Wirtschaft und Statistik 2015/4: 18-38 [https://www.destatis.de/DE/Publikationen/WirtschaftStatistik/2015/04/ ErmittlungEinwohnerzahlen_042015.pdf?_blob=publicationFile, 25.07.2016]. 
Kibele, Eva; Scholz, Rembrandt D.; Shkolnikov, Vladimir M. 2008: Low migrant mortality in Germany for men aged 65 and older: Fact or artifact? In: European Journal of Epidemiology 23,6: 389-393 [doi: 10.1007/s10654-008-9247-1].

Manke, Matthias 2005: Die Volkszählungen des Großherzogtums Mecklenburg-Schwerin in den Jahren 1819 und 1867 unter Berücksichtigung der Volkszählung des Jahres 1866. In: Genealogie 54: 449-468.

Michel, Bernd 2004: Ergebnisse der Mehrfachprüfung im Rahmen des Zensustests. In: Wirtschaft und Statistik 2004/9: 978-991 [https://www.destatis.de/DE/Publikationen/WirtschaftStatistik/Zensus/MehrFachfallPruefung.pdf?_blob=publicationFile, 25.07.2016].

Michel, Harald 1985: Volkszählungen in Deutschland. Die Erfassung des Bevölkerungsstandes von 1816 bis 1933. In: Jahrbuch für Wirtschaftsgeschichte 1985/2: 79-91.

Oettel, Andreas 2006: Zur Geschichte der amtlichen Statistik in der Sowjetischen Besatzungszone und der DDR 1945-1990. In: Statistik in Sachsen 1): 49-55.

Pötzsch, Olga 2016: Fertility in Germany before and after the 2011 Census: Still no Trend Reversal in Sight. In: Comparative Population Studies 41,1: 87-118 [doi: 10.12765/ CPoS-2016-02en].

Prewitt, Kenneth 2003: Politics and Science in Census Taking. New York, Washington: Russell Sage Foundation.

Rendtel, Ulrich 2015: Warum im Zensus die Ergebnisse der Stichprobenmethode keine Benachteiligung der großen Gemeinden darstellen. Eine Detektivarbeit. Fachbereich Wirtschaftswissenschaft; Diskussionsbeiträge Economics 2015/24 [https:/github. com/DerSpiegel/datenlese/tree/master/Zensus 2011] [http://edocs.fu-berlin.de/docs/ servlets/MCRFileNodeServlet/FUDOCS_derivate_000000005297/discpaper2015_24. pdf; jsessionid=BBBC272A205E6C2CA2C̄5432A97D84F26?hosts =, 22.08.2016].

Rennefanz, Sabine; Zylka, Regine 2013: Zensus: Wowereit bezweifelt Zensus-Daten. Berliner Zeitung vom 14. Juni 2013 [http://www.berliner-zeitung.de/berlin/zensus-wowereit-bezweifelt-zensus-daten-5464458].

Rothenbacher, Franz 1997: Historische Haushalts- und Familienstatistik von Deutschland 1815-1990. Frankfurt/New York: Campus Verlag.

Scholz, Rembrandt D. 2013: Zur demographischen Entwicklung der Hansestadt Rostock im 19. Jahrhundert. In: Busch, Michael; Kroll, Stefan; Scholz, Rembrandt D. (Hrsg.): Geschichte - Kartographie - Demographie: Historisch-Geographische Informationssysteme im methodischen Vergleich. Münster: LIT Verlag.

Statistische Ämter des Bundes und der Länder 2004: Ergebnisse des Zensustests. In: Wirtschaft und Statistik 8/2004: 813-833 [https://www.destatis.de/DE/Publikationen/ WirtschaftStatistik/Zensus/Test_Ergebnisse.pdf?_blob=publicationFile, 25.07.2016].

Statistisches Bundesamt 1999: DDR-Statistik. Grundlagen, Methoden und Organisation der amtlichen Statistik der DDR. 1949-1990. Sonderreihe mit Beiträgen für das Gebiet der ehemaligen DDR. Wiesbaden: Statistisches Bundesamt.

Statistisches Bundesamt 2013a: Bevölkerung und Erwerbstätigkeit. Natürliche Bevölkerungsbewegung. Fachserie 1 Reihe 1.1 2011. Statistisches Bundesamt (9. April 2013). Pflegestatistik 2011, Deutschlandergebnisse (18. Januar 2013).

Statistisches Bundesamt 2013b: Gemeindeverzeichnis-Sonderveröffentlichung. Gebietsstand: 31.12.2011 (Jahr). Alle politisch selbständigen Gemeinden in Deutschland nach Bevölkerung am 31.12.2011 auf Grundlage des Zensus 2011 und früherer Zählungen. Aktualisiert auf Zensusdaten mit dem Stand vom 10.04.2014 im April 2014. Wiesbaden. 
Statistisches Bundesamt 2014a: Downloadtabelle „Bevölkerung nach Alter in Jahren und Geschlecht für Gemeinden. Ergebnisse des Zensus am 9. Mai 2011. Version 1.04.2014".

Statistisches Bundesamt 2014b: Downloadtabelle „Bevölkerung nach Staat der Staatsangehörigkeit und Geschlecht für Gemeinden. Ergebnisse des Zensus am 9. Mai 2011. Version 1.04.2014".

Statistisches Bundesamt 2014c: Ergebnisse des Zensus 2011 [https://www.zensus2011. de/SharedDocs/Aktuelles/Ergebnisse/DemografischeGrunddaten.html?nn=3065474, 20.10.2015].

Statistisches Bundesamt 2015: Zensus 2011. Methoden und Verfahren. Wiesbaden: Statistisches Bundesamt [https://www.destatis.de/DE/Publikationen/Thematisch/Bevoelkerung/Zensus/ZensusBuLaMethodenVerfahren5121105119004.pdf;jsessionid=A92 71602B2171604AFD6094A7197CAD2.cae1?_blob=publicationFile, 25.07.2016].

Statistisches Bundesamt 2016: Zu den Klagen und Widerspruchsverfahren der Kommunen [https://www.zensus2011.de/SharedDocs/Aktuelles/KlagenUndWiderspruchsverfahren.html, 25.07.2016].

Vorndran, Ingeborg 2004: Zensustest-Verfahren und Ergebnisse der Haushaltegenerierung. In: Wirtschaft und Statistik 2004/11: 1245-1255 [https://www.destatis. de/DE/Publikationen/WirtschaftStatistik/Zensus/VerfahrenErgebnisse.pdf? blob= publicationFile; 25.07.2016].

Wietog, Jutta 2001: Volkszählung unter dem Nationalsozialismus. Berlin: Duncker und Humblot.

Zahn, Friedrich 1900: Die praktische Bedeutung der deutschen Volkszählung. In: Jahrbücher für Nationalökonomie und Statistik 20: 577-592.

ZensG 2011 Zensusgesetz (ZensG 2011) in der Fassung vom 8. Juli 2009 [https:// www.zensus2011.de/SharedDocs/Downloads/DE/Gesetze/Zensusgesetz_2011.html, 25.07.2016].

Zhang, Li-Chun; Hendriks, Coen 2012: Micro integration of register-based census data for dwelling and household. United Nations Economic Commission for Europe. Conference of European Statisticians. Oslo, Norwegen, 24-26. September 2012.

zur Nieden, Felix; Sommer, Bettina 2016: Life Expectancy in Germany Based on the 2011 Census: Was the Healthy Migrant Effect Merely an Artefact? In: Comparative Population Studies 41,2: 145-174 [doi: 10.12765/CPoS-2016-05en]. 
Eine Übersetzung dieses begutachteten und von den Autoren autorisierten deutschen Originaltextes durch das Bundesinstitut für Bevölkerungsforschung ist unter dem Titel "The Register-based Census in Germany: Historical Context and Relevance for Population Research", DOI 10.12765/ CPOS-2016-08en bzw. URN urn:nbn:de:bib-cpos-2016-08en8, auf http://www.comparativepopulationstudies. de verfügbar.

Dr. Rembrandt Scholz. Max-Planck-Institut für demografische Forschung. Rostock, Deutschland. E-Mail: scholz@demogr.mpg.de

URL: http://www.demogr.mpg.de/de/institut/mitarbeiter_1899/rembrandt_d_scholz_663. htm

Prof. Dr. Michaela Kreyenfeld ( $₫)$. Hertie School of Governance. Berlin, Deutschland. E-Mail: kreyenfeld@hertie-school.org

URL: https://www.hertie-school.org/de/fakultaet-forschung/fakultaet/profile/michaelakreyenfeld/ 


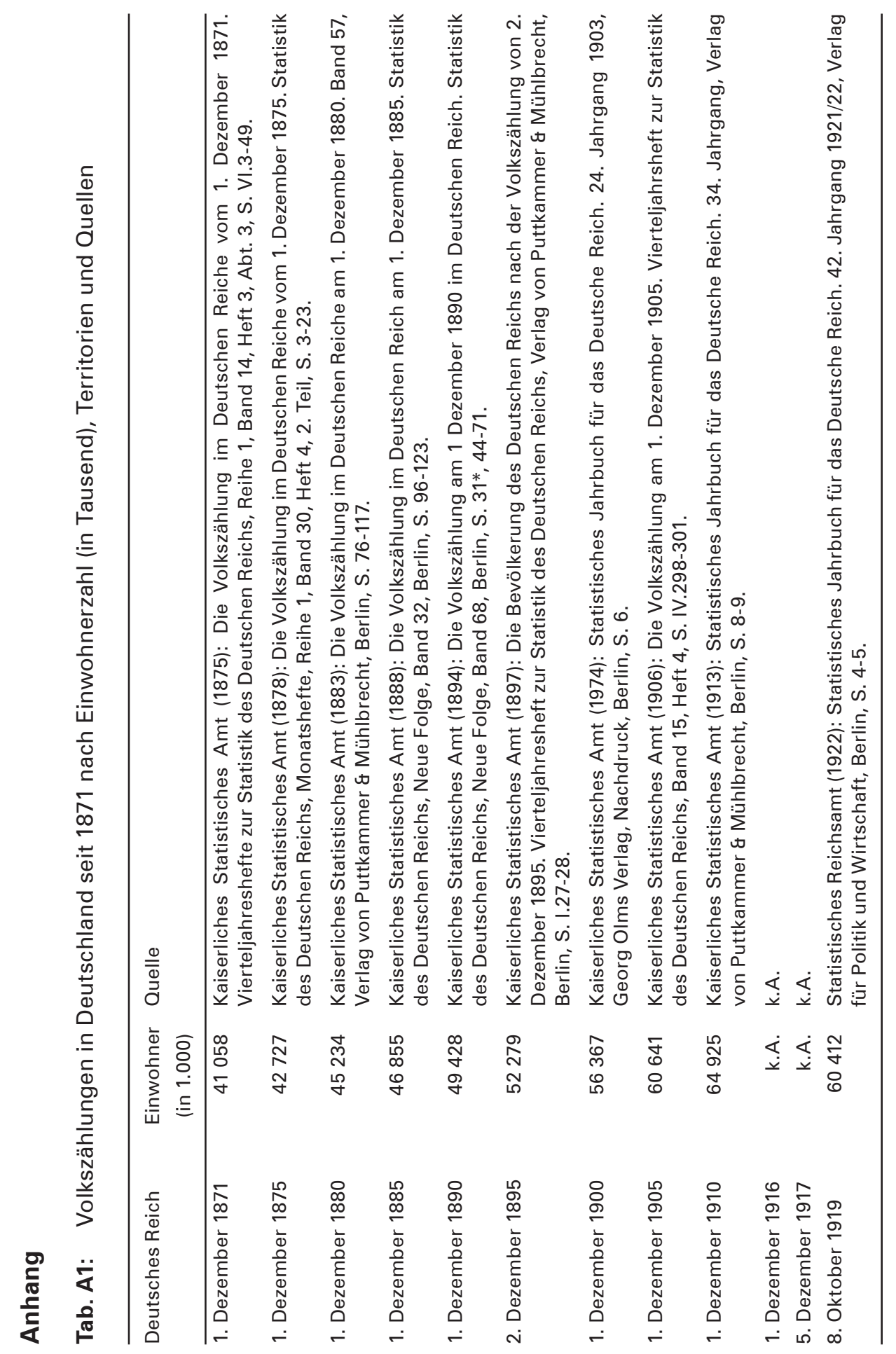


Der registergestützte Zensus in Deutschland 27

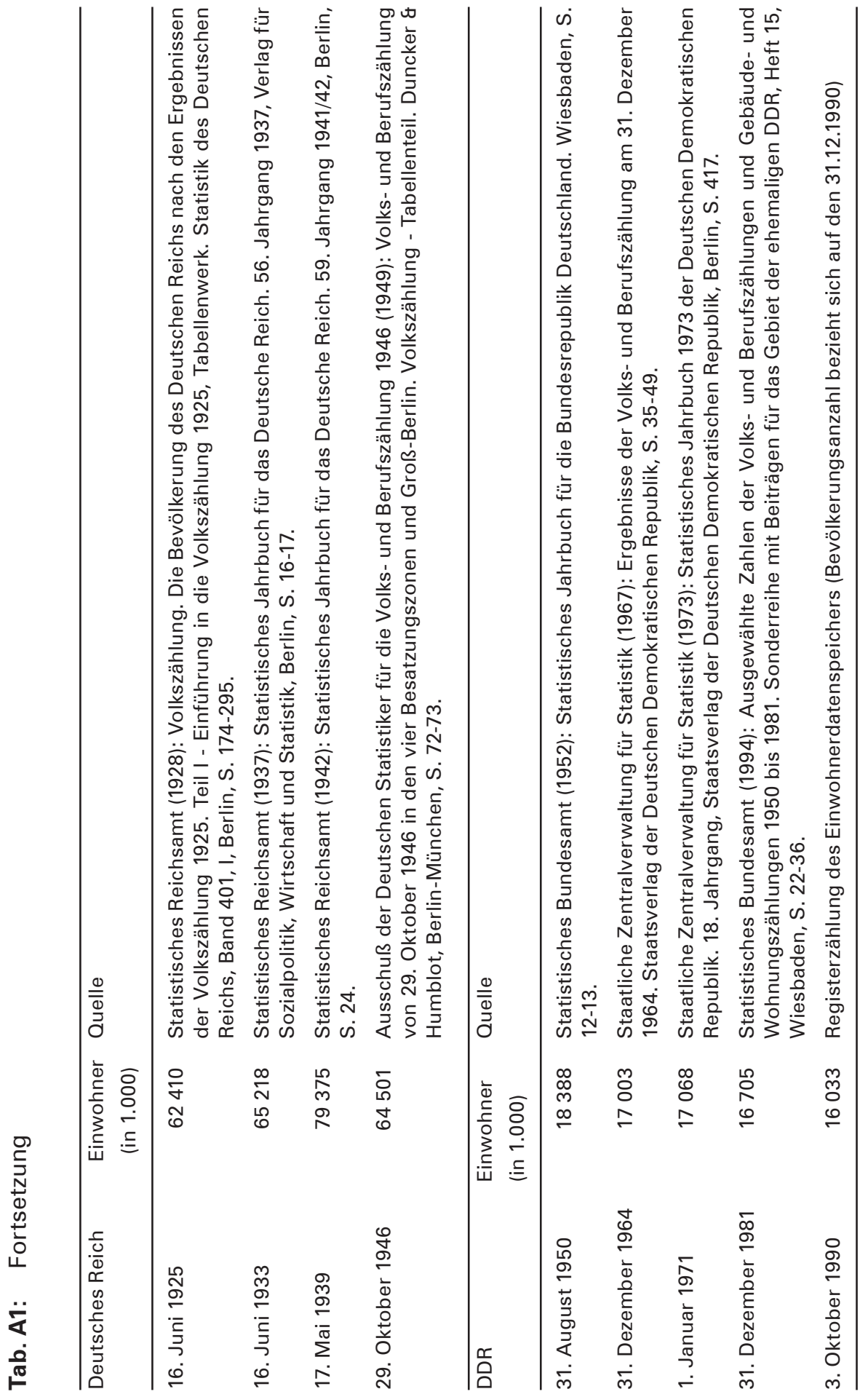




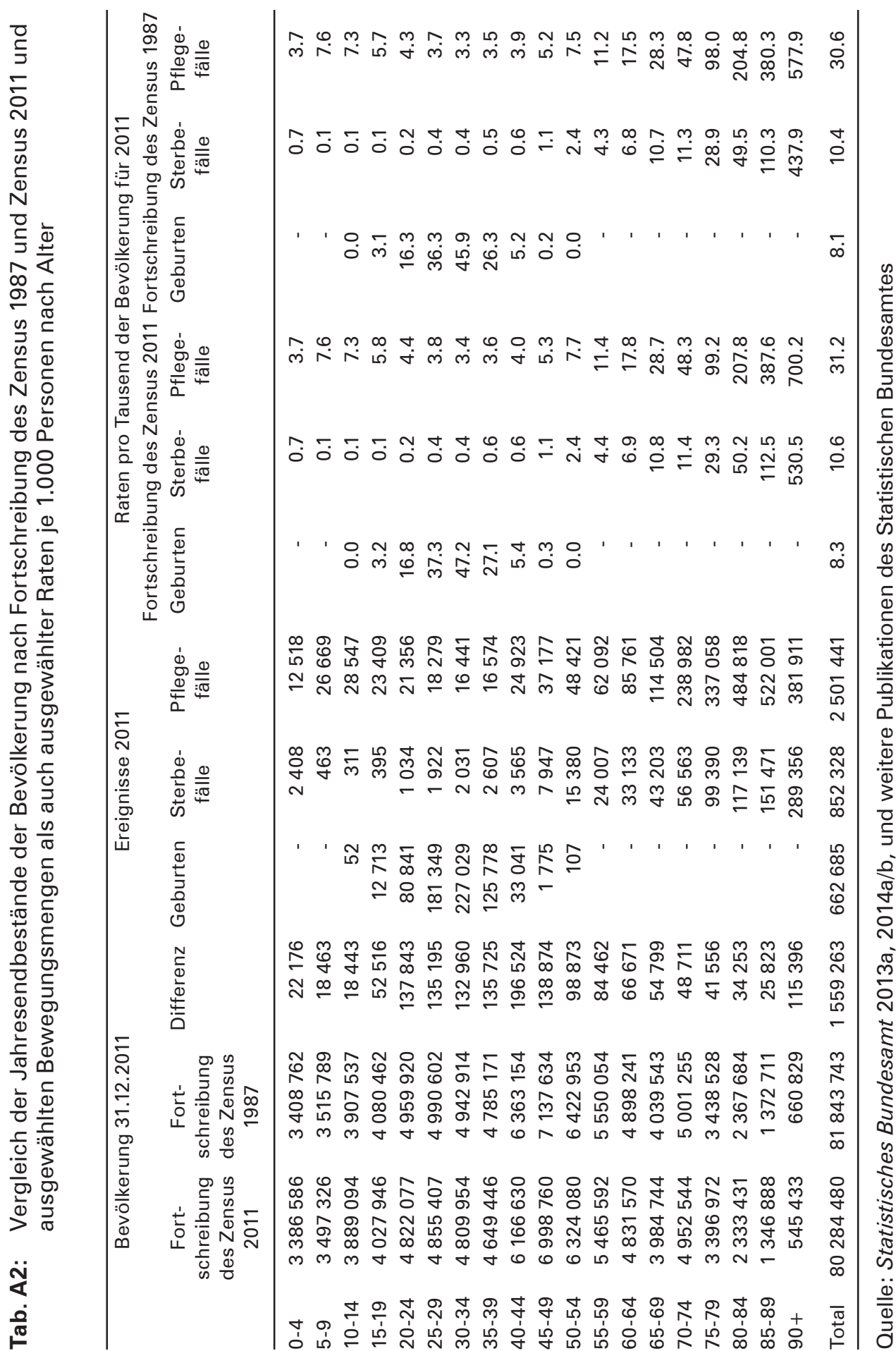


Abb. A1: Anzahl der Gemeinden (Ordinate) nach relativer Differenz zwischen Zensus 2011 und Fortschreibung auf Basis des Zensus 1987 (Abszisse), nach kleinen Gemeinden (unter 10.000 Einwohner) und großen

Gemeinden (10.000 und mehr Einwohner) und Bundesländern, in \%

Baden-Württemberg

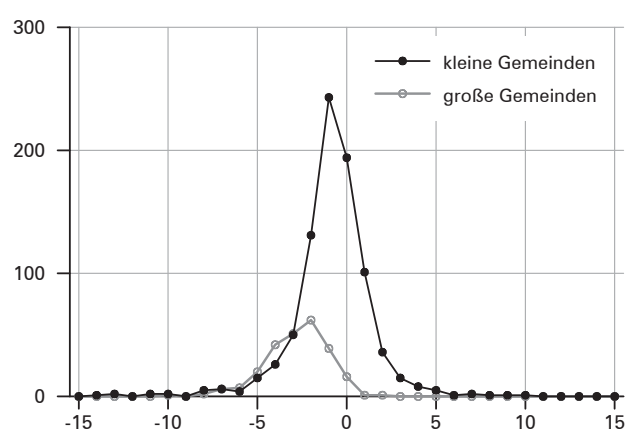

Brandenburg

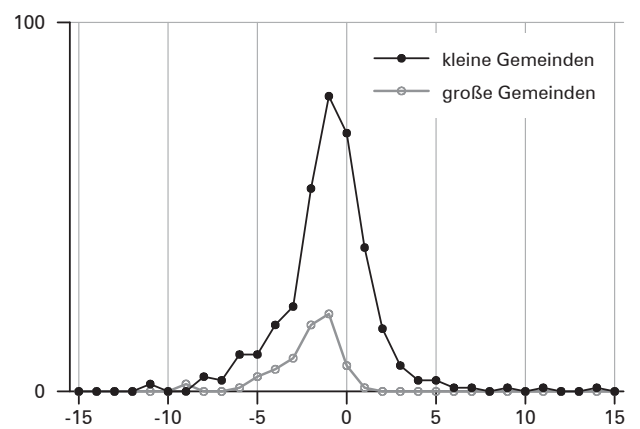

Mecklenburg-Vorpommern

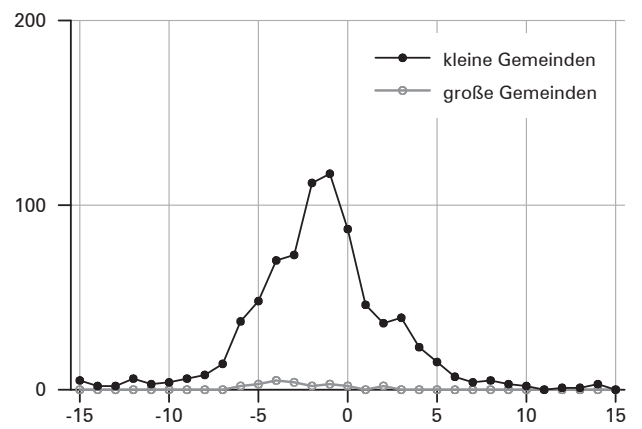

Bayern

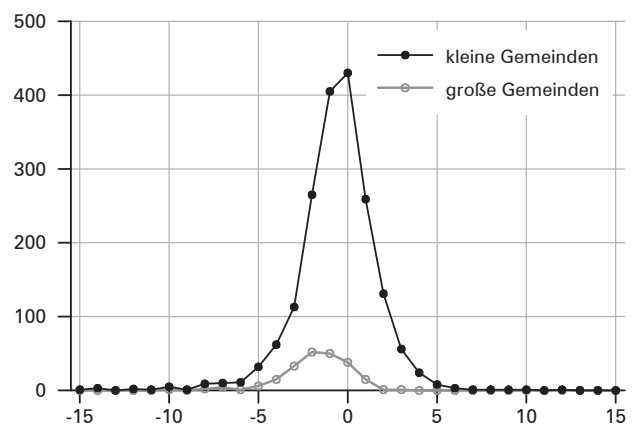

Hessen

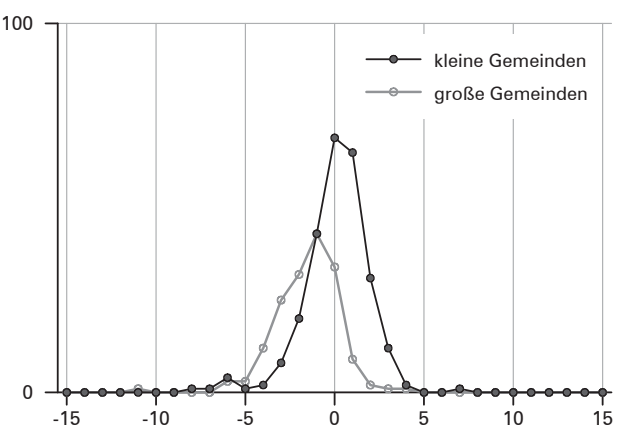

Niedersachsen

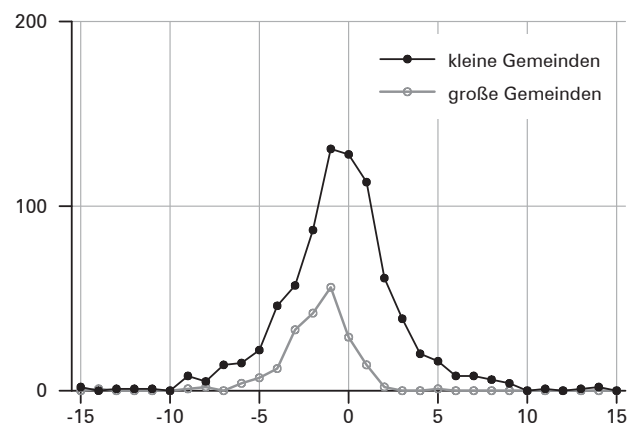




\section{Abb. A1: Fortsetzung}

Nordrhein-Westfalen

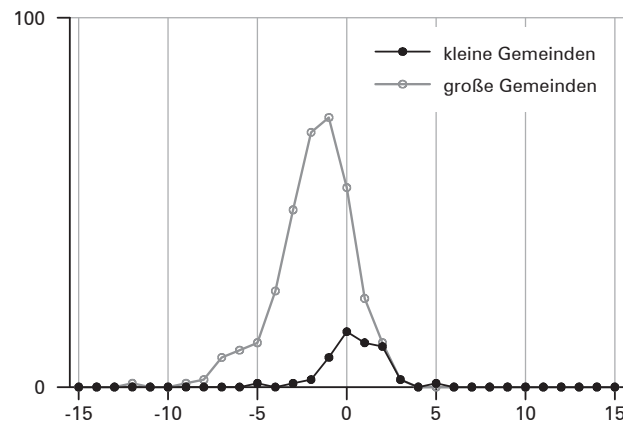

Sachsen

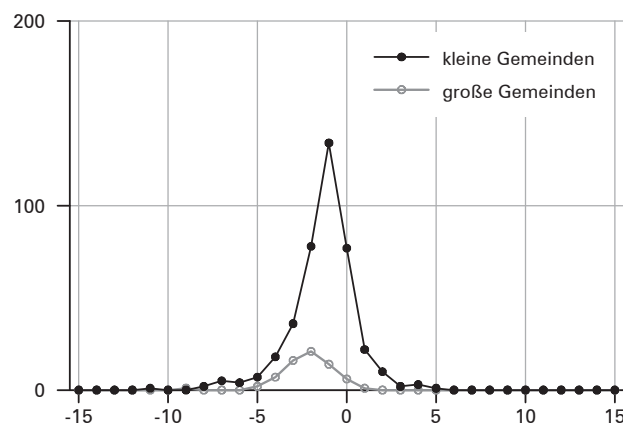

Schleswig-Holstein

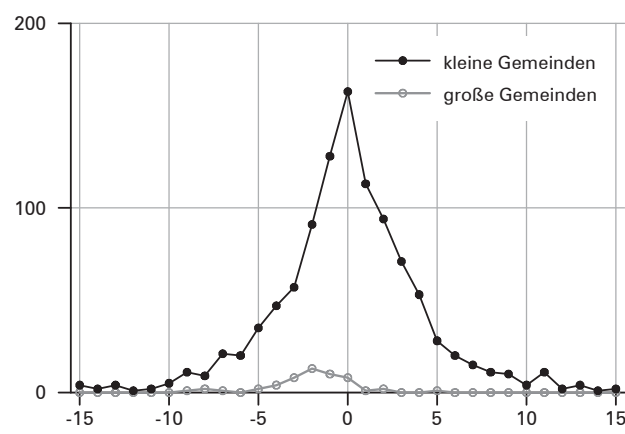

Rheinland-Pfalz

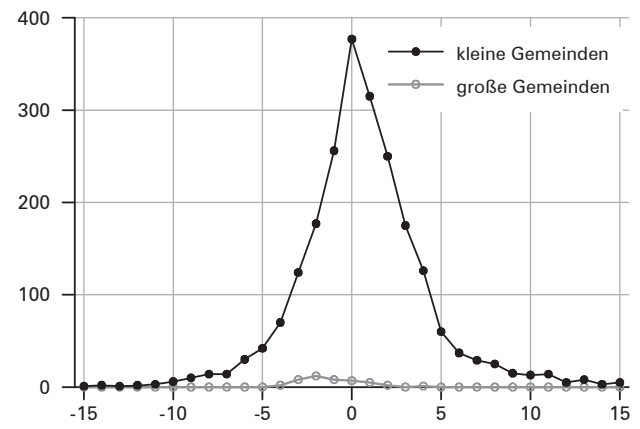

Sachsen-Anhalt

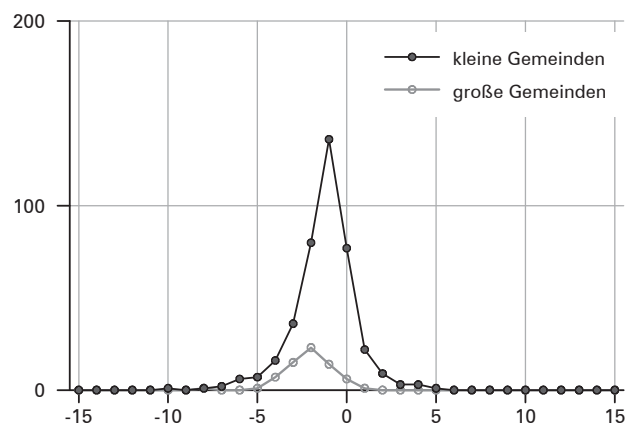

Thüringen

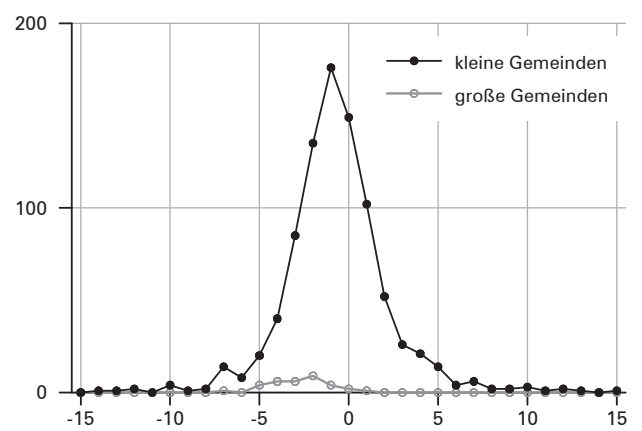

Anmerkungen: Berlin, Hamburg und Bremen wurden aus der Darstellung ausgeschlossen, da es für Stadtstaaten keine Variation nach Gemeinden gibt. Saarland wurde ausgeschlossen, da die Anzahl der Gemeinden für dieses Bundesland gering ist.

Quelle: Statistisches Bundesamt 2013b 
Abb. A2: Relative Differenz zwischen Zensus 2011 und Fortschreibung des Zensus 1987, nach Geschlecht, Alter und Staatsangehörigkeit
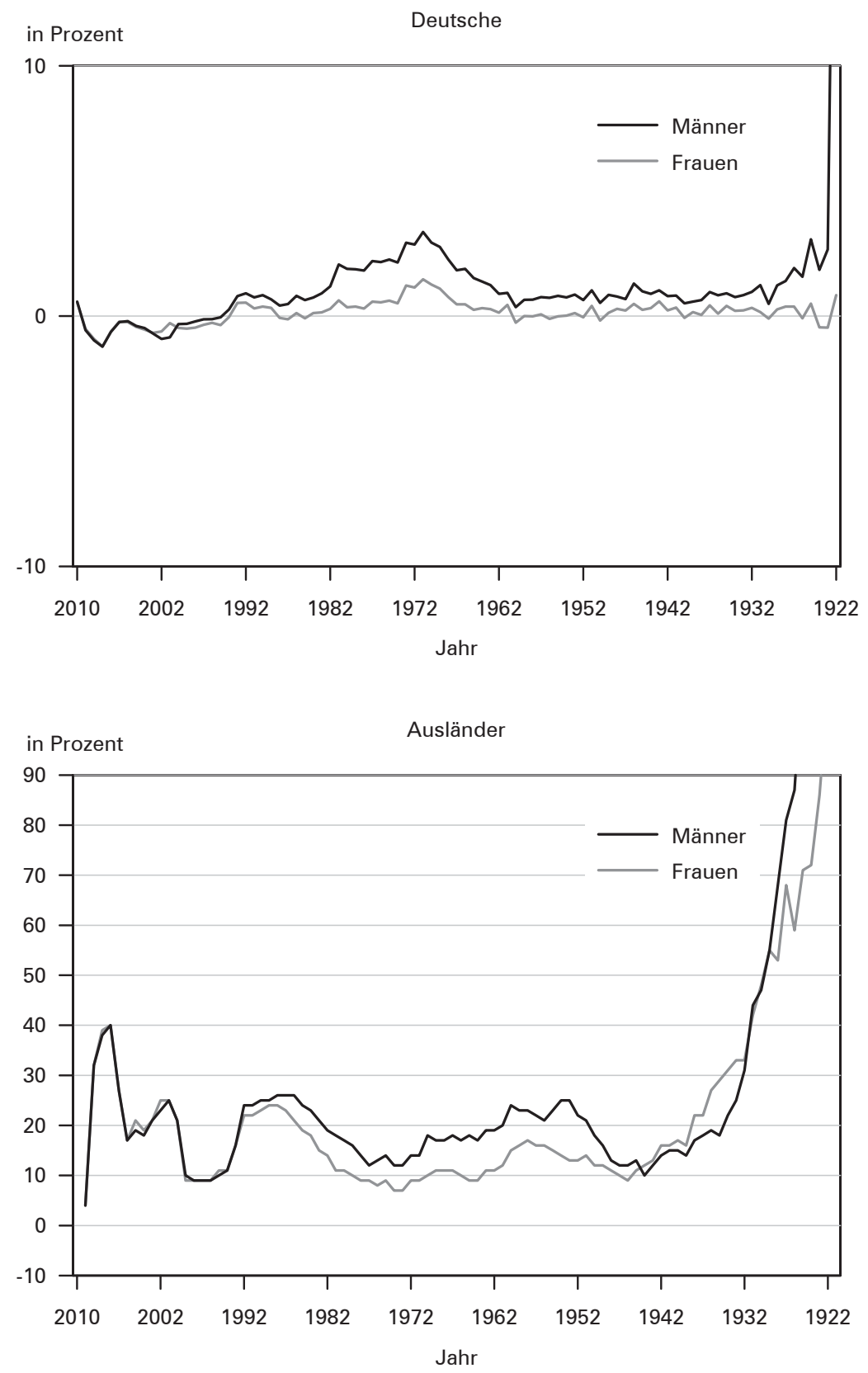

Quelle: Statistisches Bundesamt 2014 a/b 
Abb. A3: Anzahl der Personen an allen Personen (in 1.000) (Ordinate) nach Alter (Abszisse) und Gemeindegröße

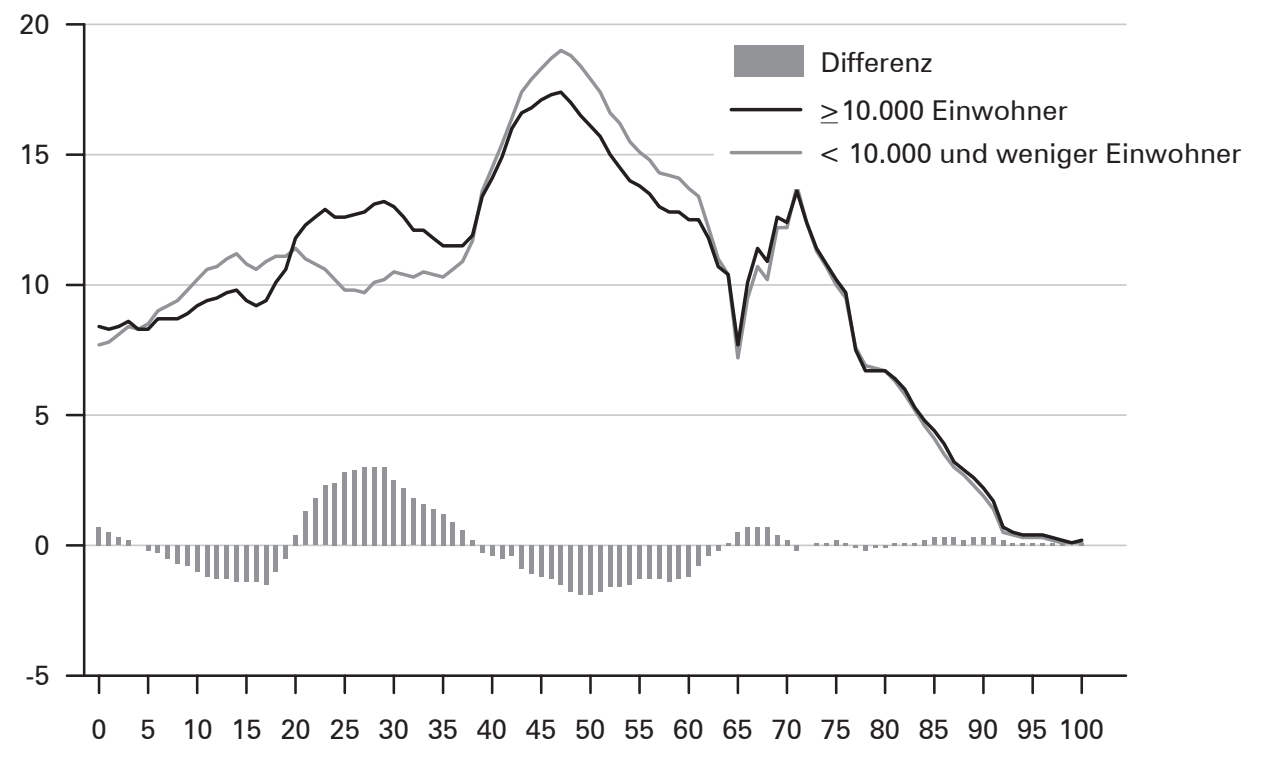

Quelle: Statistisches Bundesamt 2014b 


\section{Comparative Population Studies}

wWW.comparativepopulationstudies.de

ISSN: 1869-8980 (Print) - 1869-8999 (Internet)

\section{Published by}

Prof. Dr. Norbert F. Schneider

Federal Institute for Population Research D-65180 Wiesbaden / Germany

\section{(cc) BY-SA}

2017

\section{Managing Editor}

Frank Swiaczny

\section{Assistant Managing Editor}

Katrin Schiefer

\section{Copy Editor}

(Selected Articles in German)

Dr. Evelyn Grünheid

\section{Layout}

Beatriz Feiler-Fuchs

E-mail: cpos@bib.bund.de

\section{Scientific Advisory Board}

Paul Gans (Mannheim)

Karsten Hank (Cologne)

Johannes Huinink (Bremen)

Michaela Kreyenfeld (Rostock)

Marc Luy (Vienna)

Notburga Ott (Bochum)

Peter Preisendörfer (Mainz)

Nikola Sander (Groningen)

Zsolt Spéder (Budapest)

\section{Board of Reviewers}

Martin Abraham (Erlangen)

Laura Bernardi (Lausanne)

Hansjörg Bucher (Bonn)

Claudia Diehl (Konstanz)

Andreas Diekmann (Zurich)

Gabriele Doblhammer-Reiter (Rostock)

Jürgen Dorbritz (Wiesbaden)

Anette Eva Fasang (Berlin)

E.-Jürgen Flöthmann (Bielefeld)

Alexia Fürnkranz-Prskawetz (Vienna)

Beat Fux (Salzburg)

Joshua Goldstein (Berkeley)

Sonja Haug (Regensburg)

Hill Kulu (Liverpool)

Aart C. Liefbroer (The Hague)

Kurt Lüscher (Konstanz)

Emma Lundholm (Umeå)

Nadja Milewski (Rostock)

Dimiter Philipov (Vienna)

Roland Rau (Rostock)

Tomáš Sobotka (Vienna)

Jeroen Spijker (Barcelona)

Olivier Thévenon (Paris)

Helga de Valk (Brussels)

Heike Trappe (Rostock)

Michael Wagner (Cologne) 